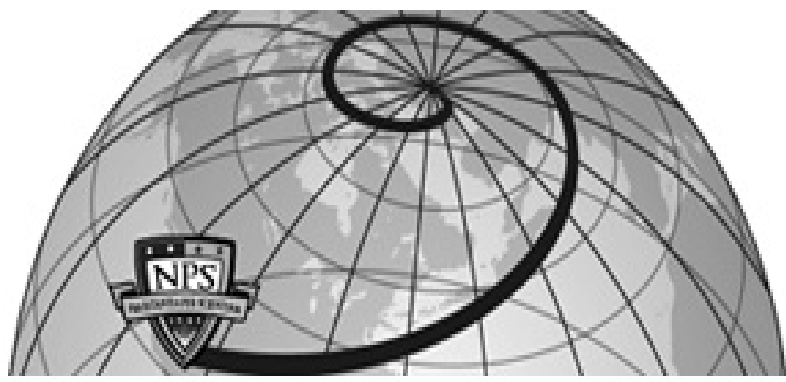

Calhoun: The NPS Institutional Archive DSpace Repository

\title{
An analysis of a new family of eighth-order optimal methods
}

Chun, Changbum; Neta, Beny

Applied Mathematics and Computation, Volume 245, (2014), pp. 86107 http://hdl.handle.net/10945/43664

This publication is a work of the U.S. Government as defined in Title 17, United States Code, Section 101. Copyright protection is not available for this work in the United States.

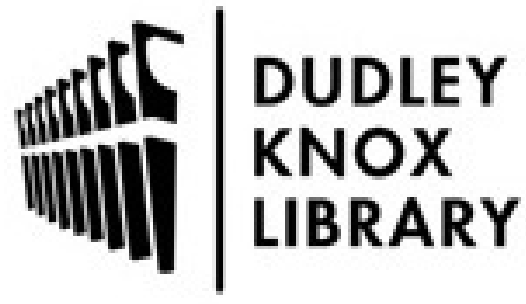

http://www.nps.edu/library
Calhoun is the Naval Postgraduate School's public access digital repository for research materials and institutional publications created by the NPS community. Calhoun is named for Professor of Mathematics Guy K. Calhoun, NPS's first appointed -- and published -- scholarly author.

Dudley Knox Library / Naval Postgraduate School 411 Dyer Road / 1 University Circle Monterey, California USA 93943 


\section{An analysis of a new family of eighth-order optimal methods}

\section{Changbum Chun ${ }^{\mathrm{a}}$, Beny Neta ${ }^{\mathrm{b}, *}$}

${ }^{a}$ Department of Mathematics, Sungkyunkwan University, Suwon 440-746, Republic of Korea

${ }^{\mathrm{b}}$ Naval Postgraduate School, Department of Applied Mathematics, Monterey, CA 93943, United States

\section{A R T I C L E I N F O}

\section{Keywords:}

Newton's method

Iterative methods

Nonlinear equations

Order of convergence

Extraneous fixed points

Basin of attraction

\begin{abstract}
A B S T R A C T
A new family of eighth order optimal methods is developed and analyzed. Numerical experiments show that our family of methods perform well and in many cases some members are superior to other eighth order optimal methods. It is shown how to choose the parameters to widen the basin of attraction.
\end{abstract}

Published by Elsevier Inc.

\section{Introduction}

There are many multistep methods for the solution of nonlinear equations, see e.g. Traub [1], and the recent book by Petković et al. [2]. The idea of optimality in such methods was introduced by Kung and Traub [3] who also developed optimal multistep method of increasing order. For example, the fourth-order optimal method given in [3] is

$$
\left\{\begin{array}{l}
w_{n}=x_{n}-\frac{f\left(x_{n}\right)}{f^{\prime}\left(x_{n}\right)}, \\
x_{n+1}=w_{n}-\frac{f\left(w_{n}\right)}{f^{\prime}\left(x_{n}\right)} \frac{1}{\left[1-\frac{f\left(w_{n}\right)}{f\left(x_{n}\right)}\right]^{2}} .
\end{array}\right.
$$

Based on this method Chun and Neta [4] constructed and analyzed the sixth order method

$$
\left\{\begin{array}{l}
w_{n}=x_{n}-\frac{f\left(x_{n}\right)}{f^{\prime}\left(x_{n}\right)}, \\
s_{n}=w_{n}-\frac{f\left(w_{n}\right)}{f^{\prime}\left(x_{n}\right)} \frac{1}{\left[1-\frac{f\left(w_{n}\right)}{f\left(x_{n}\right)}\right]^{2}}, \\
x_{n+1}=s_{n}-\frac{f\left(s_{n}\right)}{f^{\prime}\left(x_{n}\right)} \frac{1}{\left[1-\frac{f\left(w_{n}\right)}{f\left(x_{n}\right)}-\frac{f\left(s_{n}\right)}{f\left(x_{n}\right)}\right]^{2}} .
\end{array}\right.
$$

In this paper we will use the idea of weight function to develop a family of optimal eighth order methods and show how to choose the parameters to obtain the best basins of attraction.

\footnotetext{
* Corresponding author.

E-mail addresses: cbchun@skku.edu (C. Chun), bneta@nps.edu (B. Neta).
} 


\section{An optimal eighth-order method}

We consider here a generalization of the Chun-Neta sixth order scheme (2). The new family is constructed using the idea of weight functions. The multistep method is given by

$$
\left\{\begin{array}{l}
w_{n}=x_{n}-\frac{f\left(x_{n}\right)}{f^{\prime}\left(x_{n}\right)}, \\
s_{n}=w_{n}-\frac{f\left(w_{n}\right)}{f^{\prime}\left(x_{n}\right)} \frac{1}{\left[1-r_{n}\right]^{2}}, \\
x_{n+1}=s_{n}-\frac{f\left(s_{n}\right)}{f^{\prime}\left(x_{n}\right)} \frac{1}{\left.\left[1-H\left(r_{n}\right)\right]\left(t_{n}\right) P\left(q_{n}\right)\right]^{2}},
\end{array}\right.
$$

where $r_{n}=\frac{f\left(w_{n}\right)}{f\left(x_{n}\right)}, t_{n}=\frac{f\left(s_{n}\right)}{f\left(x_{n}\right)}, q_{n}=\frac{f\left(s_{n}\right)}{f\left(w_{n}\right)}$ and $H(r), J(t), P(q)$ are real-valued weight functions to be determined later.

For the method defined by (3), we have the following analysis of convergence.

Theorem 2.1. Let $\xi \in I$ be a simple zero in an open interval I of a sufficiently differentiable function $f: I \rightarrow \mathbb{R}$. Let $e_{n}=x_{n}-\xi$. Then the new family of methods defined by (3) is of optimal eighth-order when

$$
\begin{aligned}
& H(0) J(0) P(0)=2, \\
& H^{\prime}(0) P(0) J(0)=-1, \\
& H^{\prime \prime}(0) P(0) J(0)=-1, \\
& H^{\prime \prime \prime}(0) P(0) J(0)=3, \\
& \left|H^{(4)}(0)\right|<\infty, \\
& J^{\prime}(0)=-3 J(0) / 8, \\
& J^{\prime \prime}(0) \mid<\infty, \\
& P^{\prime}(0)=-P(0) / 4, \\
& \left|P^{\prime \prime}(0)\right|<\infty .
\end{aligned}
$$

The error at the $(n+1)$ th step, $e_{n+1}$, satisfies the relation

$$
\begin{aligned}
e_{n+1}= & c_{2}\left[\left(\frac{2 P^{\prime \prime}(0)}{P^{\prime}(0)}-\frac{1}{4}\right) c_{3}^{3}-c_{2} c_{3} c_{4}+\left(4-\frac{3 P^{\prime \prime}(0)}{P^{\prime}(0)}\right) c_{2}^{2} c_{3}^{2}+2 c_{2}^{3} c_{4}+\left(\frac{1}{3} P^{\prime}(0) J(0) H^{(4)}(0)+\frac{6 P^{\prime \prime}(0)}{P^{\prime}(0)}-\frac{29}{4}\right) c_{2}^{4} c_{3}\right. \\
& \left.+\left(\frac{1}{2}-\frac{2}{3} P^{\prime}(0) J(0) H^{(4)}(0)-\frac{4 P^{\prime \prime}(0)}{P^{\prime}(0)}\right) c_{2}^{6}\right] e_{n}^{8}+O\left(e_{n}^{9}\right),
\end{aligned}
$$

where $c_{i}$ are given by

$$
c_{i}=\frac{f^{(i)}(\xi)}{i ! f^{\prime}(\xi)}, \quad i \geqslant 1
$$

Proof. Let $e_{n}=x_{n}-\xi, e_{n}^{w}=w_{n}-\xi$ and $e_{n}^{s}=s_{n}-\xi$. Using the Taylor expansion of $f(x)$ around $x=\xi$ and taking $f(\xi)=0$ into account, we get

$$
f\left(x_{n}\right)=f^{\prime}(\xi)\left[e_{n}+c_{2} e_{n}^{2}+c_{3} e_{n}^{3}+c_{4} e_{n}^{4}+c_{5} e_{n}^{5}+c_{6} e_{n}^{6}+c_{7} e_{n}^{7}+c_{8} e_{n}^{8}+O\left(e_{n}^{9}\right)\right]
$$

and

$$
f^{\prime}\left(x_{n}\right)=f^{\prime}(\xi)\left[1+2 c_{2} e_{n}+3 c_{3} e_{n}^{2}+4 c_{4} e_{n}^{3}+5 c_{5} e_{n}^{4}+6 c_{6} e_{n}^{5}+7 c_{7} e_{n}^{6}+O\left(e_{n}^{7}\right)\right] .
$$

Dividing (6) by (7) gives

$$
\begin{aligned}
u_{n}= & \frac{f\left(x_{n}\right)}{f^{\prime}\left(x_{n}\right)} \\
= & e_{n}-c_{2} e_{n}^{2}+\left(-2 c_{3}+2 c_{2}^{2}\right) e_{n}^{3}+\left(-3 c_{4}+7 c_{2} c_{3}-4 c_{2}^{3}\right) e_{n}^{4}+\left(10 c_{2} c_{4}-4 c_{5}+6 c_{3}^{2}-20 c_{3} c_{2}^{2}+8 c_{2}^{4}\right) e_{n}^{5}+\left(17 c_{4} c_{3}\right. \\
& \left.-28 c_{4} c_{2}^{2}+13 c_{2} c_{5}-5 c_{6}-33 c_{2} c_{3}^{2}+52 c_{3} c_{2}^{3}-16 c_{2}^{5}\right) e_{n}^{6}+\left(-92 c_{3} c_{2} c_{4}+22 c_{3} c_{5}-18 c_{3}^{3}+126 c_{3}^{2} c_{2}^{2}-128 c_{3} c_{2}^{4}\right. \\
& \left.+12 c_{4}^{2}+72 c_{4} c_{2}^{3}-36 c_{5} c_{2}^{2}-6 c_{7}+16 c_{2} c_{6}+32 c_{2}^{6}\right) e_{n}^{7}+\left(-7 c_{8}-118 c_{5} c_{2} c_{3}+348 c_{4} c_{3} c_{2}^{2}+19 c_{2} c_{7}-64 c_{2} c_{4}^{2}\right. \\
& \left.+31 c_{4} c_{5}-75 c_{4} c_{3}^{2}-176 c_{4} c_{2}^{4}+92 c_{5} c_{2}^{3}+27 c_{6} c_{3}-44 c_{6} c_{2}^{2}+135 c_{2} c_{3}^{3}-408 c_{3}^{2} c_{2}^{3}+304 c_{3} c_{2}^{5}-64 c_{2}^{7}\right) e_{n}^{8}+O\left(e_{n}^{9}\right) .
\end{aligned}
$$


From (8), we have

$$
\begin{aligned}
e_{n}^{w}= & c_{2} e_{n}^{2}-\left(2 c_{2}^{2}-2 c_{3}\right) e_{n}^{3}-\left(3 c_{4}-7 c_{2} c_{3}+4 c_{2}^{3}\right) e_{n}^{4}+\left(-10 c_{2} c_{4}+4 c_{5}-6 c_{3}^{2}+20 c_{3} c_{2}^{2}-8 c_{2}^{4}\right) e_{n}^{5}+\left(-17 c_{4} c_{3}+28 c_{4} c_{2}^{2}\right. \\
& \left.-13 c_{2} c_{5}+5 c_{6}+33 c_{2} c_{3}^{2}-52 c_{3} c_{2}^{3}+16 c_{2}^{5}\right) e_{n}^{6}+\left(92 c_{3} c_{2} c_{4}-22 c_{3} c_{5}+18 c_{3}^{3}-126 c_{3}^{2} c_{2}^{2}+128 c_{3} c_{2}^{4}-12 c_{4}^{2}\right. \\
& \left.-72 c_{4} c_{2}^{3}+36 c_{5} c_{2}^{2}+6 c_{7}-16 c_{2} c_{6}-32 c_{2}^{6}\right) e_{n}^{7}+\left(64 c_{2}^{7}+7 c_{8}+118 c_{5} c_{2} c_{3}-348 c_{4} c_{3} c_{2}^{2}-19 c_{2} c_{7}+64 c_{2} c_{4}^{2}\right. \\
& \left.-31 c_{4} c_{5}+75 c_{4} c_{3}^{2}+176 c_{4} c_{2}^{4}-92 c_{5} c_{2}^{3}-27 c_{6} c_{3}+44 c_{6} c_{2}^{2}-135 c_{2} c_{3}^{3}+408 c_{3}^{2} c_{2}^{3}-304 c_{3} c_{2}^{5}\right) e_{n}^{8}+O\left(e_{n}^{9}\right) .
\end{aligned}
$$

Writing the Taylor's expansion for $f\left(w_{n}\right)$ and using (9), we obtain

$$
\begin{aligned}
f\left(w_{n}\right)= & f^{\prime}(\xi)\left[e_{n}^{w}+c_{2}\left(e_{n}^{w}\right)^{2}+c_{3}\left(e_{n}^{w}\right)^{3}+c_{4}\left(e_{n}^{w}\right)^{4}+O\left(\left(e_{n}^{w}\right)^{5}\right)\right] \\
= & f^{\prime}(\xi)\left[c_{2} e_{n}^{2}+\left(2 c_{3}-2 c_{2}^{2}\right) e_{n}^{3}+\left(3 c_{4}-7 c_{2} c_{3}+5 c_{2}^{3}\right) e_{n}^{4}+\left(-10 c_{2} c_{4}+4 c_{5}-6 c_{3}^{2}+24 c_{3} c_{2}^{2}-12 c_{2}^{4}\right) e_{n}^{5}\right. \\
& +\left(-17 c_{4} c_{3}+34 c_{4} c_{2}^{2}-13 c_{2} c_{5}+5 c_{6}+37 c_{2} c_{3}^{2}-73 c_{3} c_{2}^{3}+28 c_{2}^{5}\right) e_{n}^{6}+\left(18 c_{3}^{3}-64 c_{2}^{6}+6 c_{7}+104 c_{3} c_{2} c_{4}\right. \\
& \left.-16 c_{2} c_{6}-22 c_{3} c_{5}-160 c_{3}^{2} c_{2}^{2}+206 c_{3} c_{2}^{4}-12 c_{4}^{2}-104 c_{4} c_{2}^{3}+44 c_{5} c_{2}^{2}\right) e_{n}^{7}+\left(144 c_{2}^{7}+7 c_{8}+134 c_{5} c_{2} c_{3}\right. \\
& -455 c_{4} c_{3} c_{2}^{2}-19 c_{2} c_{7}+73 c_{2} c_{4}^{2}-31 c_{4} c_{5}+75 c_{4} c_{3}^{2}+297 c_{4} c_{2}^{4}-134 c_{5} c_{2}^{3}-27 c_{6} c_{3}+54 c_{6} c_{2}^{2}-147 c_{2} c_{3}^{3} \\
& \left.\left.+582 c_{3}^{2} c_{2}^{3}-552 c_{3} c_{2}^{5}\right) e_{n}^{8}+O\left(e_{n}^{9}\right)\right] .
\end{aligned}
$$

Dividing (10) by (7) gives

$$
\begin{aligned}
\frac{f\left(w_{n}\right)}{f^{\prime}\left(x_{n}\right)}= & c_{2} e_{n}^{2}+\left(2 c_{3}-4 c_{2}^{2}\right) e_{n}^{3}+\left(3 c_{4}-14 c_{2} c_{3}+13 c_{2}^{3}\right) e_{n}^{4}+\left(-20 c_{2} c_{4}+4 c_{5}-12 c_{3}^{2}+64 c_{3} c_{2}^{2}-38 c_{2}^{4}\right) e_{n}^{5}+\left(104 c_{2}^{5}\right. \\
& \left.+5 c_{6}-26 c_{2} c_{5}-34 c_{4} c_{3}+90 c_{4} c_{2}^{2}+103 c_{2} c_{3}^{2}-240 c_{3} c_{2}^{3}\right) e_{n}^{6}+O\left(e_{n}^{7}\right) .
\end{aligned}
$$

Dividing (10) by (6) gives

$$
\begin{aligned}
r_{n}= & \frac{f\left(w_{n}\right)}{f\left(x_{n}\right)} \\
= & c_{2} e_{n}+\left(2 c_{3}-3 c_{2}^{2}\right) e_{n}^{2}+\left(3 c_{4}-10 c_{2} c_{3}+8 c_{2}^{3}\right) e_{n}^{3}+\left(-14 c_{2} c_{4}+4 c_{5}-8 c_{3}^{2}+37 c_{3} c_{2}^{2}-20 c_{2}^{4}\right) e_{n}^{4} \\
& +\left(-22 c_{3} c_{4}+51 c_{4} c_{2}^{2}-18 c_{2} c_{5}+55 c_{2} c_{3}^{2}-118 c_{3} c_{2}^{3}+5 c_{6}+48 c_{2}^{5}\right) e_{n}^{5}+\left(6 c_{7}+150 c_{4} c_{2} c_{3}-22 c_{2} c_{6}-15 c_{4}^{2}-163 c_{4} c_{2}^{3}\right. \\
& \left.-28 c_{5} c_{3}+65 c_{5} c_{2}^{2}-252 c_{3}^{2} c_{2}^{2}+344 c_{3} c_{2}^{4}+26 c_{3}^{3}-112 c_{2}^{6}\right) e_{n}^{6}+O\left(e_{n}^{7}\right) .
\end{aligned}
$$

Using (8), (11) and (12), we find

$$
\begin{aligned}
e_{n}^{s}= & e_{n}^{w}-\frac{f\left(w_{n}\right)}{f^{\prime}\left(x_{n}\right)} \frac{1}{[1-r]^{2}} \\
= & \left(-c_{2} c_{3}+2 c_{2}^{3}\right) e_{n}^{4}+\left(-2 c_{2} c_{4}+14 c_{3} c_{2}^{2}-2 c_{3}^{2}-10 c_{2}^{4}\right) e_{n}^{5}+\left(31 c_{2}^{5}-3 c_{2} c_{5}-7 c_{4} c_{3}+21 c_{4} c_{2}^{2}+30 c_{2} c_{3}^{2}-72 c_{3} c_{2}^{3}\right) e_{n}^{6} \\
& +\left(20 c_{3}^{3}-74 c_{2}^{6}+88 c_{3} c_{2} c_{4}-4 c_{2} c_{6}-10 c_{3} c_{5}-188 c_{3}^{2} c_{2}^{2}+246 c_{3} c_{2}^{4}-6 c_{4}^{2}-100 c_{4} c_{2}^{3}+28 c_{5} c_{2}^{2}\right) e_{n}^{7}+O\left(e_{n}^{8}\right),
\end{aligned}
$$

so that, after elementary calculation,

$$
\begin{aligned}
f\left(s_{n}\right)= & f^{\prime}(\xi)\left[e_{n}^{s}+c_{2}\left(e_{n}^{s}\right)^{2}+c_{3}\left(e_{n}^{s}\right)^{3}+O\left(\left(e_{n}^{s}\right)^{4}\right)\right] \\
= & f^{\prime}(\xi)\left[\left(-c_{2} c_{3}+2 c_{2}^{3}\right) e_{n}^{4}+\left(-2 c_{2} c_{4}+14 c_{3} c_{2}^{2}-2 c_{3}^{2}-10 c_{2}^{4}\right) e_{n}^{5}+\left(31 c_{2}^{5}-3 c_{2} c_{5}-7 c_{4} c_{3}+21 c_{4} c_{2}^{2}+30 c_{2} c_{3}^{2}-72 c_{3} c_{2}^{3}\right) e_{n}^{6}\right. \\
& \left.+\left(20 c_{3}^{3}-74 c_{2}^{6}+88 c_{3} c_{2} c_{4}-4 c_{2} c_{6}-10 c_{3} c_{5}-188 c_{3}^{2} c_{2}^{2}+246 c_{3} c_{2}^{4}-6 c_{4}^{2}-100 c_{4} c_{2}^{3}+28 c_{5} c_{2}^{2}\right) e_{n}^{7}+O\left(e_{n}^{8}\right)\right] .
\end{aligned}
$$

An easy calculation then produces

$$
\begin{aligned}
\frac{f\left(s_{n}\right)}{f^{\prime}\left(x_{n}\right)}= & \left(-c_{2} c_{3}+2 c_{2}^{3}\right) e_{n}^{4}+\left(-2 c_{2} c_{4}+16 c_{3} c_{2}^{2}-2 c_{3}^{2}-14 c_{2}^{4}\right) e_{n}^{5}+\left(59 c_{2}^{5}-3 c_{2} c_{5}-7 c_{4} c_{3}+25 c_{4} c_{2}^{2}+37 c_{2} c_{3}^{2}-110 c_{3} c_{2}^{3}\right) e_{n}^{6} \\
& +\left(26 c_{3}^{3}-192 c_{2}^{6}+112 c_{3} c_{2} c_{4}-4 c_{2} c_{6}-10 c_{3} c_{5}-310 c_{3}^{2} c_{2}^{2}+508 c_{3} c_{2}^{4}-6 c_{4}^{2}-158 c_{4} c_{2}^{3}+34 c_{5} c_{2}^{2}\right) e_{n}^{7}+0\left(e_{n}^{8}\right), \\
t_{n}= & \frac{f\left(s_{n}\right)}{f\left(x_{n}\right)} \\
= & \left(-c_{2} c_{3}+2 c_{2}^{3}\right) e_{n}^{3}+\left(-2 c_{2} c_{4}-2 c_{3}^{2}+15 c_{3} c_{2}^{2}-12 c_{2}^{4}\right) e_{n}^{4}+\left(-3 c_{2} c_{5}-7 c_{3} c_{4}+33 c_{2} c_{3}^{2}-89 c_{3} c_{2}^{3}+23 c_{4} c_{2}^{2}+43 c_{2}^{5}\right) e_{n}^{5} \\
& +\left(98 c_{4} c_{2} c_{3}-4 c_{2} c_{6}-6 c_{4}^{2}-125 c_{4} c_{2}^{3}-10 c_{5} c_{3}+31 c_{5} c_{2}^{2}-236 c_{3}^{2} * c_{2}^{2}+347 c_{3} c_{2}^{4}+22 c_{3}^{3}-117 c_{2}^{6}\right) e_{n}^{6} \\
& +\left(130 c_{5} c_{2} c_{3}-651 c_{4} c_{3} c_{2}^{2}-5 c_{2} c_{7}-17 c_{5} c_{4}-162 c_{5} c_{2}^{3}-13 c_{6} c_{3}+39 c_{6} c_{2}^{2}+72 c_{2} c_{4}^{2}+95 c_{4} c_{3}^{2}+468 c_{4} c_{2}^{4}-266 c_{2} c_{3}^{3}\right. \\
& \left.+1087 c_{3}^{2} c_{2}^{3}-1042 c_{3} c_{2}^{5}+266 c_{2}^{7}\right) e_{n}^{7}+O\left(e_{n}^{8}\right)
\end{aligned}
$$

and 


$$
\begin{aligned}
q_{n}= & \frac{f\left(s_{n}\right)}{f\left(w_{n}\right)} \\
= & \left(-c_{3}+2 c_{2}^{2}\right) e_{n}^{2}+\left(-2 c_{4}+8 c_{2} c_{3}-6 c_{2}^{3}\right) e_{n}^{3}+\left(-3 c_{5}+7 c_{3}^{2}-25 c_{3} c_{2}^{2}+11 c_{2} c_{4}+9 c_{2}^{4}\right) e_{n}^{4}+\left(18 c_{3} c_{4}-4 c_{6}\right. \\
& \left.-30 c_{4} c_{2}^{2}+14 c_{2} c_{5}-32 c_{2} c_{3}^{2}+36 c_{3} c_{2}^{3}-2 c_{2}^{5}\right) e_{n}^{5}+\left(22 c_{5} c_{3}-71 c_{4} c_{2} c_{3}-5 c_{7}-36 c_{5} c_{2}^{2}+17 c_{2} c_{6}+11 c_{4}^{2}\right. \\
& \left.+37 c_{4} c_{2}^{3}-13 c_{3}^{3}+41 c_{3}^{2} c_{2}^{2}+26 c_{3} c_{2}^{4}-28 c_{2}^{6}\right) e_{n}^{6}+O\left(e_{n}^{7}\right) .
\end{aligned}
$$

We now expand $H\left(r_{n}\right), J\left(t_{n}\right), P\left(q_{n}\right)$ into Taylor series about 0 to obtain

$$
\begin{aligned}
& H\left(r_{n}\right)=H(0)+H^{\prime}(0) r_{n}+\frac{H^{\prime \prime}(0)}{2} r_{n}^{2}+\frac{H^{\prime \prime \prime}(0)}{6} r_{n}^{3}+O\left(r_{n}^{4}\right), \\
& J\left(t_{n}\right)=J(0)+J^{\prime}(0) t_{n}+\frac{J^{\prime \prime}(0)}{2} t_{n}^{2}+O\left(t_{n}^{3}\right), \\
& P\left(q_{n}\right)=P(0)+P^{\prime}(0) q_{n}+\frac{P^{\prime \prime}(0)}{2} q_{n}^{2}+O\left(q_{n}^{3}\right) .
\end{aligned}
$$

Upon using the values

$$
\begin{aligned}
& H(0) J(0) P(0)=2, \\
& H^{\prime}(0) P(0) J(0)=-1, \\
& H^{\prime \prime}(0) P(0) J(0)=-1, \\
& H^{\prime \prime \prime}(0) P(0) J(0)=3, \\
& J^{\prime}(0)=-3 J(0) / 8, \\
& P^{\prime}(0)=-P(0) / 4
\end{aligned}
$$

and Eqs. (11), (16) and (17) we obtain

$$
\begin{aligned}
\frac{1}{\left[1-H\left(r_{n}\right) J\left(t_{n}\right) P\left(q_{n}\right)\right]^{2}}= & 1+2 c_{2} e_{n}+3 c_{3} e_{n}^{2}+4 c_{4} e_{n}^{3} \\
& +\left[\left(2 \frac{P^{\prime \prime}(0)}{P^{\prime}(0)}+\frac{1}{3} P^{\prime}(0) J(0) H^{(4)}(0)-\frac{9}{4}\right) c_{2}^{4}+5 c_{5}-c_{2} c_{4}+\left(\frac{9}{2}-2 \frac{P^{\prime \prime}(0)}{P^{\prime}(0)}\right) c_{3} c_{2}^{2}+\frac{1}{2}\left(\frac{P^{\prime \prime}(0)}{P^{\prime}(0)}-\frac{1}{2}\right) c_{3}^{2}\right] e_{n}^{4} \\
& +O\left(e_{n}^{5}\right) .
\end{aligned}
$$

Therefore, from (13), (15) and (19), we obtain

$$
\begin{aligned}
e_{n+1}= & e_{n}^{s}-\frac{f\left(s_{n}\right)}{f^{\prime}\left(x_{n}\right)} \frac{1}{[1-H(r) J(t) P(q)]^{2}} \\
= & c_{2}\left[\left(\frac{2 P^{\prime \prime}(0)}{P^{\prime}(0)}-\frac{1}{4}\right) c_{3}^{3}-c_{2} c_{3} c_{4}+\left(4-\frac{3 P^{\prime \prime}(0)}{P^{\prime}(0)}\right) c_{2}^{2} c_{3}^{2}+2 c_{2}^{3} c_{4}+\left(\frac{1}{3} P^{\prime}(0) J(0) H^{(4)}(0)+\frac{6 P^{\prime \prime}(0)}{P^{\prime}(0)}-\frac{29}{4}\right) c_{2}^{4} c_{3}\right. \\
& \left.+\left(\frac{1}{2}-\frac{2}{3} P^{\prime}(0) J(0) H^{(4)}(0)-\frac{4 P^{\prime \prime}(0)}{P^{\prime}(0)}\right) c_{2}^{6}\right] e_{n}^{8}+O\left(e_{n}^{9}\right),
\end{aligned}
$$

this completing the proof.

Now we can choose

$$
\begin{aligned}
& H(r)=\frac{a+b r+c r^{2}}{1+d r+g r^{2}}, \\
& J(t)=\frac{\alpha+\beta t}{1+\gamma t}, \\
& P(q)=\frac{A+B q}{1+C q} .
\end{aligned}
$$

It is easy to see that $B=A(C-1 / 4), a=\frac{2}{A \alpha}, b=\frac{1-4 g}{A \alpha}, \beta=\alpha(\gamma-3 / 8), c=\frac{1}{2} \frac{8 g-3}{A \alpha}$, and $d=1-2 g$. Substituting these values in $H, J$ and $P$, we have

$$
\begin{aligned}
& H(r)=\frac{1}{2} \frac{4+(2-8 g) r+(8 g-3) r^{2}}{A \alpha\left(1+(1-2 g) r+g r^{2}\right)}, \\
& J(t)=\frac{1}{8} \frac{\alpha(8+(8 \gamma-3) t)}{1+\gamma t},
\end{aligned}
$$




$$
P(q)=\frac{1}{4} \frac{A(4+(4 C-1) q)}{1+C q} .
$$

Therefore we have 5 parameters $\alpha, \gamma, A, C, g$. We can choose $C=-2 / 3$ and $g=41 / 36$ by requiring that $3-4 P^{\prime}(0) J(0) H^{(4)}(0)-\frac{24 P^{\prime \prime}(0)}{P^{\prime}(0)}=0$ and $4-\frac{3 P^{\prime \prime}(0)}{P^{\prime}(0)}=0$, which eliminate the terms of $c_{2}^{6}$ and $c_{2}^{2} c_{3}^{2}$ in the error Eq. (4). Thus we have

$$
\begin{aligned}
& H(r)=\frac{2\left(36-64 r+55 r^{2}\right)}{A \alpha\left(36-46 r+41 r^{2}\right)}, \\
& J(t)=\frac{1}{8} \frac{\alpha(8+(8 \gamma-3) t)}{1+\gamma t}, \\
& P(q)=\frac{1}{4} \frac{A(11 q-12)}{2 q-3} .
\end{aligned}
$$

This family of methods is denoted by OM.

Since we only use the product $H(r) J(t) P(q)$, it is easy to see that $A \alpha$ cancels out.

$$
H(r) J(t) P(q)=\frac{1}{2} \frac{4+(2-8 g) r+(8 g-3) r^{2}}{A \alpha\left(1+(1-2 g) r+g r^{2}\right)} \frac{1}{8} \frac{\alpha(8+(8 \gamma-3) t)}{1+\gamma t} \frac{1}{4} \frac{A(4+(4 C-1) q)}{1+C q} .
$$

Thus we have the 3 free parameters $g, \gamma$ and $C$. This family is denoted OMN. Clearly OM is a special case since it has a specific choice of $g$ and $C$. In the next section we give a comparative numerical study of several members of our families OM and OMN as well as other well known optimal eighth order methods. We will also discuss the basins of attraction of our families.

\section{Numerical examples}

In this section we present some numerical experiments using our newly found methods and compare these results to other schemes. All computations were done using MAPLE using 128 digit floating point arithmetic (Digits :=128). Given an initial guess $x_{0}$ we decide that the method converges if the sequence $\left\{x_{n}\right\}$ generated by the iterative method has a residual $f\left(x_{n}\right) \mid$ less than a tolerance $\epsilon=10^{-25}$ in a maximum of 100 iterations, otherwise we consider the method to be divergent. We used the following test functions and display the approximate zero $x_{*}$ found up to the 28 th decimal places.

$\begin{array}{lll}\text { Index } & \text { Test function } & \text { Root } \\ 1 & x^{3}+4 x^{2}-10 & 1.3652300134140968457608068290 \\ 2 & \sin ^{2}(x)-x^{2}+1 & 1.4044916482153412260350868178 \\ 3 & (x-1)^{3}-1 & 2.0 \\ 4 & x^{3}-10 & 2.15443469003188372175929356665 \\ 5 & x e^{x^{2}}-\sin ^{2}(x)+3 \cos (x)+5 & -1.2076478271309189270094167584 \\ 6 & e^{x^{2}+7 x-30}-1 & 3.0 \\ 7 & \sin (x)-\frac{x}{2} & 1.8954942670339809471440357381 \\ 8 & x^{5}+x-10000 & 6.3087771299726890947675717718 \\ 9 & \sqrt{x}-\frac{1}{x}-3 & 9.6335955628326951924063127092 \\ 10 & e^{x}+x-20 & 2.8424389537844470678165859402 \\ 11 & \ln (x)+\sqrt{x}-5 & 8.3094326942315717953469556827 \\ 12 & x^{2}-e^{x}-3 x+2 & .2575302854398607604553673049 \\ 13 & e^{x} \sin (x)+\ln \left(1+x^{2}\right) & 0 \\ 14 & e^{-x^{2}+x+2}-1 & -1 \\ 15 & x^{5}+x^{4}+4 x^{2}-15 & 1.347428098968304981506715381\end{array}$

Compared to our methods OM and OMN with various parameters we have taken the following methods

- Kung-Traub eighth-order method (KT8) [3] defined by

$$
\begin{aligned}
& y_{n}=x_{n}-\frac{f\left(x_{n}\right)}{f^{\prime}\left(x_{n}\right)}, \\
& \tau_{n}=y_{n}-\frac{f\left(x_{n}\right)}{f^{\prime}\left(x_{n}\right)} \frac{f\left(y_{n}\right) f\left(x_{n}\right)}{\left[f\left(x_{n}\right)-f\left(y_{n}\right)\right]^{2}}, \\
& x_{n+1}=\tau_{n}-\frac{f\left(x_{n}\right)}{f^{\prime}\left(x_{n}\right)} \frac{f\left(x_{n}\right) f\left(y_{n}\right) f\left(\tau_{n}\right)}{\left[f\left(x_{n}\right)-f\left(y_{n}\right)\right]^{2}} \frac{f^{2}\left(x_{n}\right)+f\left(y_{n}\right)\left[f\left(y_{n}\right)-f\left(\tau_{n}\right)\right]}{\left[f\left(x_{n}\right)-f\left(\tau_{n}\right)\right]^{2}\left[f\left(y_{n}\right)-f\left(\tau_{n}\right)\right]} .
\end{aligned}
$$


- The method based on Kung-Traub optimal fourth-order method and Hermite interpolating polynomial (HKT8) [2]

$$
\begin{aligned}
& y_{n}=x_{n}-\frac{f\left(x_{n}\right)}{f^{\prime}\left(x_{n}\right)}, \\
& \tau_{n}=y_{n}-\frac{f\left(y_{n}\right)}{f^{\prime}\left(x_{n}\right)} \frac{1}{\left[1-f\left(y_{n}\right) / f\left(x_{n}\right)\right]^{2}}, \\
& x_{n+1}=\tau_{n}-\frac{f\left(\tau_{n}\right)}{H_{3}^{\prime}\left(\tau_{n}\right)},
\end{aligned}
$$

where

$$
H_{3}^{\prime}\left(\tau_{n}\right)=2\left(f\left[x_{n}, \tau_{n}\right]-f\left[x_{n}, y_{n}\right]\right)+f\left[y_{n}, \tau_{n}\right]+\frac{y_{n}-\tau_{n}}{y_{n}-x_{n}}\left(f\left[x_{n}, y_{n}\right]-f^{\prime}\left(x_{n}\right)\right) .
$$

- The method based on Kung-Traub optimal fourth-order method and Hermite interpolating polynomial replacing the function (HKN8) [5]

$$
\begin{aligned}
& y_{n}=x_{n}-\frac{f\left(x_{n}\right)}{f^{\prime}\left(x_{n}\right)}, \\
& \tau_{n}=y_{n}-\frac{f\left(y_{n}\right)}{f^{\prime}\left(x_{n}\right)} \frac{1}{\left[1-f\left(y_{n}\right) / f\left(x_{n}\right)\right]^{2}}, \\
& x_{n+1}=\tau_{n}-\frac{H_{3}\left(\tau_{n}\right)}{f^{\prime}\left(\tau_{n}\right)},
\end{aligned}
$$

\begin{tabular}{|c|c|c|c|c|c|c|c|c|}
\hline$f$ & & KT8 & НКT8 & HKN8 & N8 & WM8 & OM1 & OM2 \\
\hline $\begin{array}{l}f_{1} \\
x_{0}=1.5\end{array}$ & $\begin{array}{l}\text { IT } \\
f\left(x_{*}\right)\end{array}$ & $\begin{array}{l}3 \\
3.5 e-69\end{array}$ & $\begin{array}{l}3 \\
4.8 \mathrm{e}-72\end{array}$ & $\begin{array}{l}3 \\
4.8 \mathrm{e}-72\end{array}$ & $\begin{array}{l}3 \\
1 \mathrm{e}-66\end{array}$ & $\begin{array}{l}3 \\
2.3 e-60\end{array}$ & $\begin{array}{l}3 \\
-5.4 \mathrm{e}-86\end{array}$ & $\begin{array}{l}3 \\
-5.7 e-83\end{array}$ \\
\hline $\begin{array}{l}f_{2} \\
x_{0}=1.37\end{array}$ & $\begin{array}{l}\text { IT } \\
f\left(x_{*}\right)\end{array}$ & $\begin{array}{l}3 \\
-1.1 \mathrm{e}-90\end{array}$ & $\begin{array}{l}3 \\
-4.4 \mathrm{e}-94\end{array}$ & $\begin{array}{l}3 \\
-9.7 e-59\end{array}$ & $\begin{array}{l}3 \\
-1.6 e-86\end{array}$ & $\begin{array}{l}3 \\
-2.1 \mathrm{e}-80\end{array}$ & $\begin{array}{l}3 \\
1.1 \mathrm{e}-97\end{array}$ & $\begin{array}{l}3 \\
1.3 e-97\end{array}$ \\
\hline $\begin{array}{l}f_{3} \\
x_{0}=2.5\end{array}$ & $\begin{array}{l}\text { IT } \\
f\left(x_{*}\right)\end{array}$ & $\begin{array}{l}4 \\
0\end{array}$ & $\begin{array}{l}3 \\
9.5 e-26\end{array}$ & $\begin{array}{l}3 \\
9.5 e-26\end{array}$ & $\begin{array}{l}4 \\
0\end{array}$ & $\begin{array}{l}4 \\
0\end{array}$ & $\begin{array}{l}3 \\
-3.7 e-29\end{array}$ & $\begin{array}{l}3 \\
-1.6 e-31\end{array}$ \\
\hline $\begin{array}{l}f_{4} \\
x_{0}=4\end{array}$ & $\begin{array}{l}\text { IT } \\
f\left(x_{*}\right)\end{array}$ & $\begin{array}{l}4 \\
1.1 \mathrm{e}-116\end{array}$ & $\begin{array}{l}4 \\
0\end{array}$ & $\begin{array}{l}4 \\
0\end{array}$ & $\begin{array}{l}4 \\
2.2 e-104\end{array}$ & $\begin{array}{l}4 \\
6.4 e-83\end{array}$ & $\begin{array}{l}4 \\
0\end{array}$ & $\begin{array}{l}4 \\
0\end{array}$ \\
\hline $\begin{array}{l}f_{5} \\
x_{0}=-1.5\end{array}$ & $\begin{array}{l}\text { IT } \\
f\left(x_{*}\right)\end{array}$ & $\begin{array}{l}4 \\
-1.2 \mathrm{e}-126\end{array}$ & $\begin{array}{l}4 \\
-1.2 \mathrm{e}-126\end{array}$ & $\begin{array}{l}4 \\
2.6 e-101\end{array}$ & $\begin{array}{l}4 \\
-1.2 \mathrm{e}-126\end{array}$ & $\begin{array}{l}4 \\
-1.2 \mathrm{e}-126\end{array}$ & $\begin{array}{l}3 \\
3.5 e-37\end{array}$ & $\begin{array}{l}3 \\
1.6 e-28\end{array}$ \\
\hline $\begin{array}{l}f_{6} \\
x_{0}=4\end{array}$ & $\begin{array}{l}\text { IT } \\
f\left(x_{*}\right)\end{array}$ & $\begin{array}{l}8 \\
3.7 e-40\end{array}$ & $\begin{array}{l}9 \\
0\end{array}$ & $\begin{array}{l}8 \\
-4.8 \mathrm{e}-73\end{array}$ & $\begin{array}{l}9 \\
0\end{array}$ & $\begin{array}{l}9 \\
1.6 e-33\end{array}$ & $\begin{array}{l}8 \\
-2 e-126\end{array}$ & $\begin{array}{l}7 \\
-2.2 \mathrm{e}-42\end{array}$ \\
\hline $\begin{array}{l}f_{7} \\
x_{0}=2\end{array}$ & $\begin{array}{l}\text { IT } \\
f\left(x_{*}\right)\end{array}$ & $\begin{array}{l}3 \\
-4,4 e-71\end{array}$ & $\begin{array}{l}3 \\
-3 e-76\end{array}$ & $\begin{array}{l}3 \\
-1.4 \mathrm{e}-49\end{array}$ & $\begin{array}{l}3 \\
-3.6 e-68\end{array}$ & $\begin{array}{l}3 \\
-1.2 \mathrm{e}-63\end{array}$ & $\begin{array}{l}3 \\
2.4 \mathrm{e}-84\end{array}$ & $\begin{array}{l}3 \\
2.1 \mathrm{e}-86\end{array}$ \\
\hline $\begin{array}{l}f_{8} \\
x_{0}=4\end{array}$ & $\begin{array}{l}\text { IT } \\
f\left(x_{*}\right)\end{array}$ & $\begin{array}{l}5 \\
1 e-63\end{array}$ & $\begin{array}{l}5 \\
0\end{array}$ & $\begin{array}{l}5 \\
-4.9 e-85\end{array}$ & $\begin{array}{l}5 \\
8.3 e-47\end{array}$ & $\begin{array}{l}101 \\
9.8 \mathrm{e} 17\end{array}$ & $\begin{array}{l}15 \\
0\end{array}$ & $\begin{array}{l}42 \\
-7 e-124\end{array}$ \\
\hline $\begin{array}{l}f_{9} \\
x_{0}=1\end{array}$ & $\begin{array}{l}\text { IT } \\
f\left(x_{*}\right)\end{array}$ & $\begin{array}{l}4 \\
-1 \mathrm{e}-127\end{array}$ & $\begin{array}{l}4 \\
2.9 e-89\end{array}$ & $\begin{array}{l}5 \\
1.5 e-70\end{array}$ & $\begin{array}{l}4 \\
0\end{array}$ & $\begin{array}{l}4 \\
-1.4 \mathrm{e}-71\end{array}$ & $\begin{array}{l}4 \\
0\end{array}$ & $\begin{array}{l}4 \\
1 \mathrm{e}-127\end{array}$ \\
\hline $\begin{array}{l}f_{10} \\
x_{0}=2.1\end{array}$ & $\begin{array}{l}\text { IT } \\
f\left(x_{*}\right)\end{array}$ & $\begin{array}{l}4 \\
0\end{array}$ & $\begin{array}{l}4 \\
0\end{array}$ & $\begin{array}{l}4 \\
-1.4 \mathrm{e}-112\end{array}$ & $\begin{array}{l}4 \\
2.4 \mathrm{e}-117\end{array}$ & $\begin{array}{l}7 \\
3.8 e-29\end{array}$ & $\begin{array}{l}4 \\
-2 e-126\end{array}$ & $\begin{array}{l}4 \\
0\end{array}$ \\
\hline $\begin{array}{l}f_{11} \\
x_{0}=1\end{array}$ & $\begin{array}{l}\text { IT } \\
f\left(x_{*}\right)\end{array}$ & $\begin{array}{l}3 \\
1.8 \mathrm{e}-34\end{array}$ & $\begin{array}{l}4 \\
-2.9 e-106\end{array}$ & $\begin{array}{l}4 \\
2.5 e-29\end{array}$ & $\begin{array}{l}3 \\
9.2 e-30\end{array}$ & $\begin{array}{l}4 \\
-1.1 \mathrm{e}-96\end{array}$ & $\begin{array}{l}4 \\
-1 \mathrm{e}-127\end{array}$ & $\begin{array}{l}4 \\
0\end{array}$ \\
\hline $\begin{array}{l}f_{12} \\
x_{0}=0.5\end{array}$ & $\begin{array}{l}\text { IT } \\
f\left(x_{*}\right)\end{array}$ & $\begin{array}{l}3 \\
1.2 \mathrm{e}-84\end{array}$ & $\begin{array}{l}3 \\
4.1 \mathrm{e}-87\end{array}$ & $\begin{array}{l}3 \\
3.9 e-51\end{array}$ & $\begin{array}{l}3 \\
4.5 e-86\end{array}$ & $\begin{array}{l}3 \\
5.3 e-84\end{array}$ & $\begin{array}{l}3 \\
6 e-84\end{array}$ & $\begin{array}{l}3 \\
6.1 e-84\end{array}$ \\
\hline $\begin{array}{l}f_{13} \\
x_{0}=1\end{array}$ & $\begin{array}{l}\text { IT } \\
f\left(x_{*}\right)\end{array}$ & $\begin{array}{l}4 \\
9.4 e-67\end{array}$ & $\begin{array}{l}4 \\
5.8 \mathrm{e}-80\end{array}$ & $\begin{array}{l}4 \\
9.9 e-55\end{array}$ & $\begin{array}{l}4 \\
5.4 e-60\end{array}$ & $\begin{array}{l}4 \\
7.4 e-49\end{array}$ & $\begin{array}{l}4 \\
-1.1 \mathrm{e}-93\end{array}$ & $\begin{array}{l}4 \\
-4.9 e-107\end{array}$ \\
\hline $\begin{array}{l}f_{14} \\
x_{0}=-0.85\end{array}$ & $\begin{array}{l}\text { IT } \\
f\left(x_{*}\right)\end{array}$ & $\begin{array}{l}3 \\
2.3 e-46\end{array}$ & $\begin{array}{l}3 \\
3.2 e-49\end{array}$ & $\begin{array}{l}3 \\
6.4 e-34\end{array}$ & $\begin{array}{l}3 \\
2.3 e-43\end{array}$ & $\begin{array}{l}3 \\
3.2 e-38\end{array}$ & $\begin{array}{l}4 \\
-1.9 e-83\end{array}$ & $\begin{array}{l}4 \\
-5.8 \mathrm{e}-83\end{array}$ \\
\hline $\begin{array}{l}f_{15} \\
x_{0}=1.2\end{array}$ & $\begin{array}{l}\text { IT } \\
f\left(x_{*}\right)\end{array}$ & $\begin{array}{l}3 \\
1.4 e-43\end{array}$ & $\begin{array}{l}3 \\
2.9 e-46\end{array}$ & $\begin{array}{l}3 \\
-6.9 e-33\end{array}$ & $\begin{array}{l}3 \\
9.4 e-38\end{array}$ & $\begin{array}{l}3 \\
5 e-31\end{array}$ & $\begin{array}{l}3 \\
-1.1 \mathrm{e}-52\end{array}$ & $\begin{array}{l}3 \\
-4.7 e-49\end{array}$ \\
\hline
\end{tabular}

Table 1

Comparison of eighth-order iterative schemes. 
Table 2

Comparison of eighth-order iterative schemes.

\begin{tabular}{|c|c|c|c|c|c|}
\hline$f$ & & OMN1 & OMN2 & OMN3 & OMN6 \\
\hline $\begin{array}{l}f_{1} \\
x_{0}=1.5\end{array}$ & $\begin{array}{l}\text { IT } \\
f\left(x_{*}\right)\end{array}$ & $\begin{array}{l}3 \\
-1.0 e-62\end{array}$ & $\begin{array}{l}3 \\
-8.9 e-67\end{array}$ & $\begin{array}{l}3 \\
-2.4 e-75\end{array}$ & $\begin{array}{l}3 \\
-4.1-68\end{array}$ \\
\hline $\begin{array}{l}f_{2} \\
x_{0}=1.37\end{array}$ & $\begin{array}{l}\text { IT } \\
f\left(x_{*}\right)\end{array}$ & $\begin{array}{l}3 \\
9.5 e-82\end{array}$ & $\begin{array}{l}3 \\
1.6 e-83\end{array}$ & $\begin{array}{l}3 \\
5.1 \mathrm{e}-92\end{array}$ & $\begin{array}{l}3 \\
9.6 e-89\end{array}$ \\
\hline $\begin{array}{l}f_{3} \\
x_{0}=2.5\end{array}$ & $\begin{array}{l}\text { IT } \\
f\left(x_{*}\right)\end{array}$ & $\begin{array}{l}4 \\
-8.9 e-121\end{array}$ & $\begin{array}{l}3 \\
-6.4 e-30\end{array}$ & $\begin{array}{l}3 \\
-1.7 e-29\end{array}$ & $\begin{array}{l}4 \\
-3.0 e-127\end{array}$ \\
\hline $\begin{array}{l}f_{4} \\
x_{0}=4\end{array}$ & $\begin{array}{l}\text { IT } \\
f\left(x_{*}\right)\end{array}$ & $\begin{array}{l}4 \\
-2.1 \mathrm{e}-35\end{array}$ & $\begin{array}{l}4 \\
0\end{array}$ & $\begin{array}{l}4 \\
-1.4 \mathrm{e}-126\end{array}$ & $\begin{array}{l}4 \\
-7.8 \mathrm{e} 94\end{array}$ \\
\hline $\begin{array}{l}f_{5} \\
x_{0}=-1.5\end{array}$ & $\begin{array}{l}\text { IT } \\
f\left(x_{*}\right)\end{array}$ & $\begin{array}{l}4 \\
5.5 e-105\end{array}$ & $\begin{array}{l}4 \\
-1.1 \mathrm{e}-126\end{array}$ & $\begin{array}{l}4 \\
1.2 \mathrm{e}-126\end{array}$ & $\begin{array}{l}4 \\
-9.8 e-75\end{array}$ \\
\hline $\begin{array}{l}f_{6} \\
x_{0}=4\end{array}$ & $\begin{array}{l}\text { IT } \\
f\left(x_{*}\right)\end{array}$ & $\begin{array}{l}10 \\
-6.2 e-46\end{array}$ & $\begin{array}{l}8 \\
-2.0 e-126\end{array}$ & $\begin{array}{l}6 \\
-1.2 \mathrm{e}-38\end{array}$ & $\begin{array}{l}10 \\
0\end{array}$ \\
\hline $\begin{array}{l}f_{7} \\
x_{0}=2\end{array}$ & $\begin{array}{l}\text { IT } \\
f\left(x_{*}\right)\end{array}$ & $\begin{array}{l}3 \\
2.8 e-66\end{array}$ & $\begin{array}{l}3 \\
1.6 e-71\end{array}$ & $\begin{array}{l}3 \\
1.0 e-78\end{array}$ & $\begin{array}{l}3 \\
2.2 \mathrm{e}-68\end{array}$ \\
\hline $\begin{array}{l}f_{8} \\
x_{0}=4\end{array}$ & $\begin{array}{l}\text { IT } \\
f\left(x_{*}\right)\end{array}$ & $\begin{array}{l}6 \\
0\end{array}$ & $\begin{array}{l}5 \\
-4.0 e-72\end{array}$ & $\begin{array}{l}5 \\
-2.1 \mathrm{e}-96\end{array}$ & $\begin{array}{l}101 \\
-5.5 e 20\end{array}$ \\
\hline $\begin{array}{l}f_{9} \\
x_{0}=1\end{array}$ & $\begin{array}{l}\text { IT } \\
f\left(x_{*}\right)\end{array}$ & $\begin{array}{l}5 \\
2.0 \mathrm{e}-111\end{array}$ & $\begin{array}{l}4 \\
-1.1 \mathrm{e}-52\end{array}$ & $\begin{array}{l}4 \\
-3.8 e-82\end{array}$ & $\begin{array}{l}4 \\
1.8 \mathrm{e}-84\end{array}$ \\
\hline $\begin{array}{l}f_{10} \\
x_{0}=2.1\end{array}$ & $\begin{array}{l}\text { IT } \\
f\left(x_{*}\right)\end{array}$ & $\begin{array}{l}4 \\
-1.9 e-124\end{array}$ & $\begin{array}{l}4 \\
0\end{array}$ & $\begin{array}{l}4 \\
-3.3 e-76\end{array}$ & $\begin{array}{l}4 \\
-2.1 \mathrm{e}-121\end{array}$ \\
\hline $\begin{array}{l}f_{11} \\
x_{0}=1\end{array}$ & $\begin{array}{l}\text { IT } \\
f\left(x_{*}\right)\end{array}$ & $\begin{array}{l}4 \\
6.2 e-90\end{array}$ & $\begin{array}{l}4 \\
2.2 e-109\end{array}$ & $\begin{array}{l}4 \\
0\end{array}$ & $\begin{array}{l}4 \\
-9.3 e-45\end{array}$ \\
\hline $\begin{array}{l}f_{12} \\
x_{0}=0.5\end{array}$ & $\begin{array}{l}\text { IT } \\
f\left(x_{*}\right)\end{array}$ & $\begin{array}{l}3 \\
2.4 \mathrm{e}-78\end{array}$ & $\begin{array}{l}3 \\
1.1 \mathrm{e}-86\end{array}$ & $\begin{array}{l}3 \\
1.5 e-87\end{array}$ & $\begin{array}{l}3 \\
1.5 e-78\end{array}$ \\
\hline $\begin{array}{l}f_{13} \\
x_{0}=1\end{array}$ & $\begin{array}{l}\text { IT } \\
f\left(x_{*}\right)\end{array}$ & $\begin{array}{l}4 \\
-4.3 e-32\end{array}$ & $\begin{array}{l}4 \\
-1.3 e-69\end{array}$ & $\begin{array}{l}4 \\
-8.7 e-86\end{array}$ & $\begin{array}{l}4 \\
-1.1 \mathrm{e}-34\end{array}$ \\
\hline $\begin{array}{l}f_{14} \\
x_{0}=-0.85\end{array}$ & $\begin{array}{l}\text { IT } \\
f\left(x_{*}\right)\end{array}$ & $\begin{array}{l}4 \\
-2.5 e-64\end{array}$ & $\begin{array}{l}4 \\
-8.6 e-116\end{array}$ & $\begin{array}{l}4 \\
-4.6 e-124\end{array}$ & $\begin{array}{l}4 \\
-1.7 e-73\end{array}$ \\
\hline $\begin{array}{l}f_{15} \\
x_{0}=1.2\end{array}$ & $\begin{array}{l}\text { IT } \\
f\left(x_{*}\right)\end{array}$ & $\begin{array}{l}3 \\
-1.9 e-36\end{array}$ & $\begin{array}{l}3 \\
-6.1 e-35\end{array}$ & $\begin{array}{l}3 \\
-7.6 e-40\end{array}$ & $\begin{array}{l}3 \\
-2.7 e-41\end{array}$ \\
\hline
\end{tabular}

Table 3

The parameters for each member. The first 2 belong to OM and the last 6 are OMN members.

\begin{tabular}{llrl}
\hline Case & $g$ & $\gamma$ & $C$ \\
\hline 1 & $41 / 36$ & 2 & $-2 / 3$ \\
2 & $41 / 36$ & -1 & $-2 / 3$ \\
3 & -4 & 0 & -4 \\
4 & -4 & 0 & 0 \\
5 & 0 & -4 & 0 \\
6 & 0 & 0 & -4 \\
7 & 0 & 0 & 0 \\
8 & 2.9 & -4 & -4 \\
\hline
\end{tabular}

where

$$
H_{3}\left(\tau_{n}\right)=f\left(x_{n}\right)+f^{\prime}\left(x_{n}\right) \frac{\left(\tau_{n}-y_{n}\right)^{2}\left(\tau_{n}-x_{n}\right)}{\left(y_{n}-x_{n}\right)\left(x_{n}+2 y_{n}-3 \tau_{n}\right)}+f^{\prime}\left(\tau_{n}\right) \frac{\left(\tau_{n}-y_{n}\right)\left(x_{n}-\tau_{n}\right)}{x_{n}+2 y_{n}-3 \tau_{n}}-f\left[x_{n}, y_{n}\right] \frac{\left(\tau_{n}-x_{n}\right)^{3}}{\left(y_{n}-x_{n}\right)\left(x_{n}+2 y_{n}-3 \tau_{n}\right)} .
$$

- An eighth order (N8) optimal method proposed by Neta [6] and based on King's fourth order optimal method [7] with $\beta=2$ given by

$$
\begin{aligned}
& y_{n}=x_{n}-\frac{f\left(x_{n}\right)}{f^{\prime}\left(x_{n}\right)}, \\
& \tau_{n}=y_{n}-\frac{f\left(y_{n}\right)}{f^{\prime}\left(x_{n}\right)} \frac{f\left(x_{n}\right)+\beta f\left(y_{n}\right)}{f\left(x_{n}\right)+(\beta-2) f\left(y_{n}\right)}, \\
& x_{n+1}=x_{n}-\frac{f\left(x_{n}\right)}{f^{\prime}\left(x_{n}\right)}+\gamma f^{2}\left(x_{n}\right)-\rho f^{3}\left(x_{n}\right),
\end{aligned}
$$


Table 4

Number of EFPs, minimum and maximum values of the absolute value of the real parts of EFPs.

\begin{tabular}{lllc}
\hline Case & Number of EFPs & Min. value & Max. value \\
\hline 1 & 54 & $5.06 \mathrm{e}-2$ & 0.507 \\
2 & 54 & $8.6 \mathrm{e}-3$ & 0.353 \\
3 & 48 & $9.7 \mathrm{e}-5$ & 3.038 \\
4 & 48 & $1 . \mathrm{e}-8$ & 0.838 \\
5 & 48 & $2.85 \mathrm{e}-2$ & 0.524 \\
6 & 48 & $1.85 \mathrm{e}-2$ & 3.040 \\
7 & 48 & $1.96 \mathrm{e}-3$ & 0.495 \\
8 & 54 & 0.161 & 11.364 \\
\hline
\end{tabular}

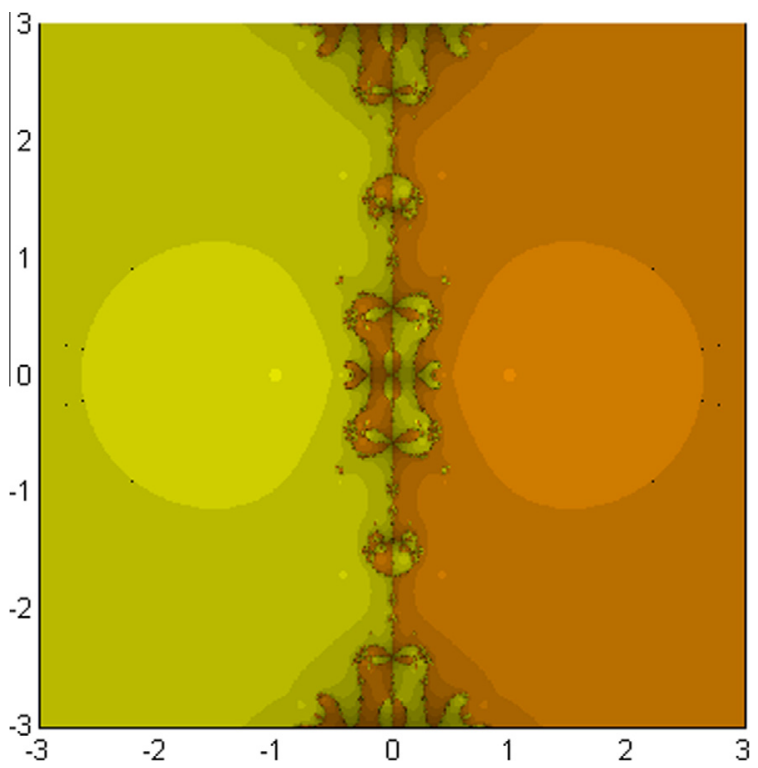

Fig. 1. Our method with $g=41 / 36, \gamma=2, C=-2 / 3, \alpha=1$, and $A=-3$ for the roots of the polynomial $z^{2}-1$.

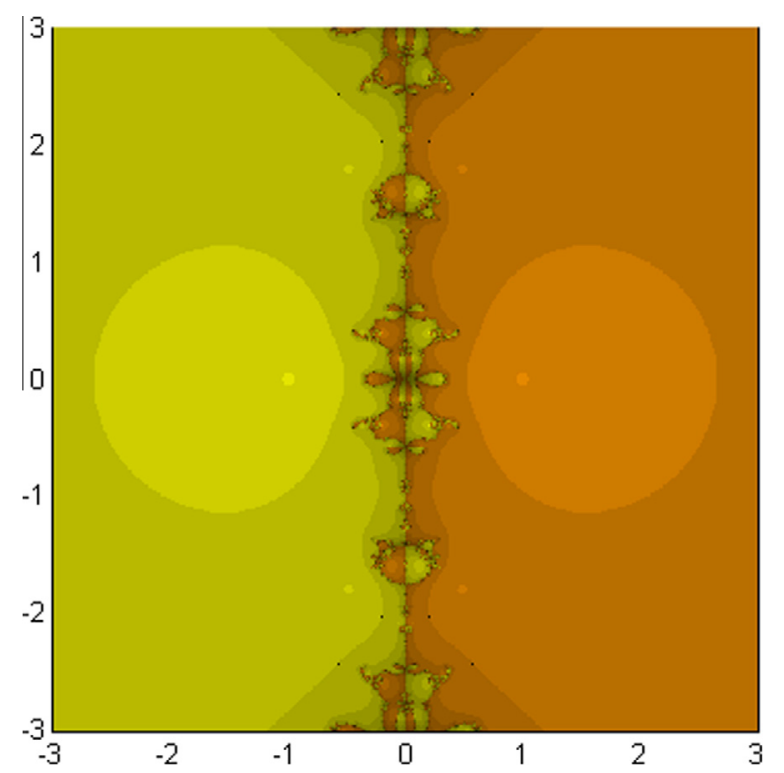

Fig. 2. Our method with $g=41 / 36, \gamma=-1, C=-2 / 3, \alpha=2$, and $A=1$ for the roots of the polynomial $z^{2}-1$. 


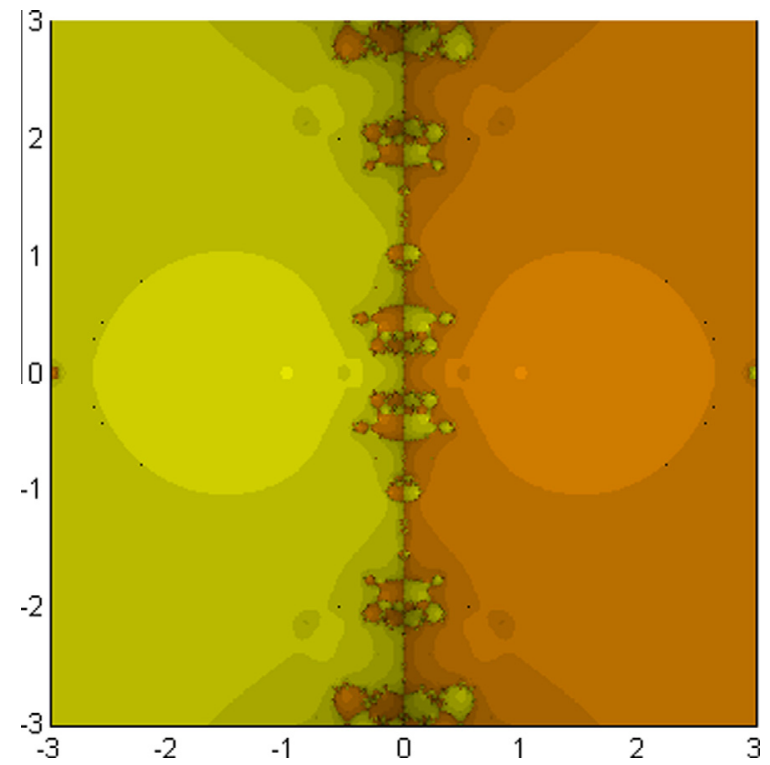

Fig. 3. Our method with $g=-4, \gamma=0, C=-4$, and any $\alpha$ and $A$ for the roots of the polynomial $z^{2}-1$.

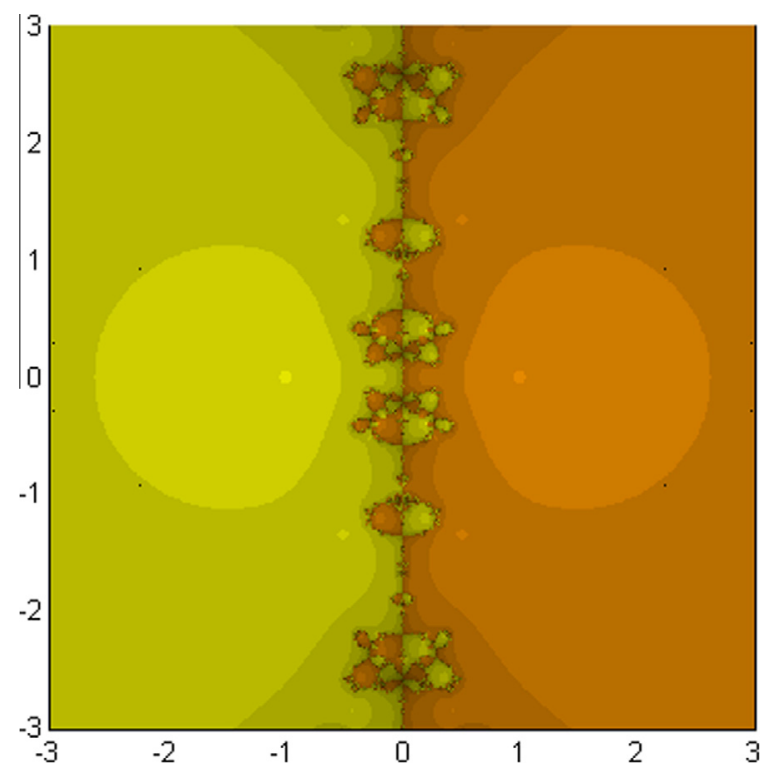

Fig. 4. Our method with $g=-4, \gamma=0, C=0$, and any $\alpha$ and $A$ for the roots of the polynomial $z^{2}-1$.

where

$$
\begin{aligned}
& \rho=\frac{\phi_{y}-\phi_{t}}{F_{y}-F_{t}}, \quad \gamma=\phi_{y}-\rho F_{y}, \quad F_{y}=f\left(y_{n}\right)-f\left(x_{n}\right), \quad F_{t}=f\left(\tau_{n}\right)-f\left(x_{n}\right), \\
& \phi_{y}=\frac{y_{n}-x_{n}}{F_{y}^{2}}-\frac{1}{F_{y} f^{\prime}\left(x_{n}\right)}, \quad \phi_{t}=\frac{\tau_{n}-x_{n}}{F_{t}^{2}}-\frac{1}{F_{t} f^{\prime}\left(x_{n}\right)} .
\end{aligned}
$$

- A weight function based eighth order (WM8) optimal method [2] (using the fourth order Maheshwari's method [8]) given by 


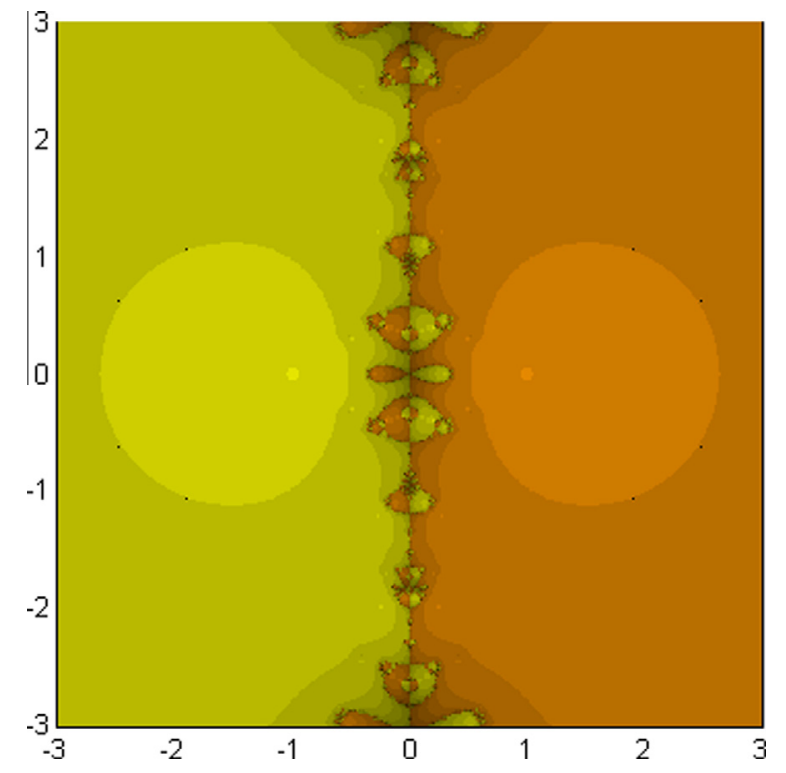

Fig. 5. Our method with $g=0, \gamma=-4, C=0$, and any $\alpha$ and $A$ for the roots of the polynomial $z^{2}-1$.

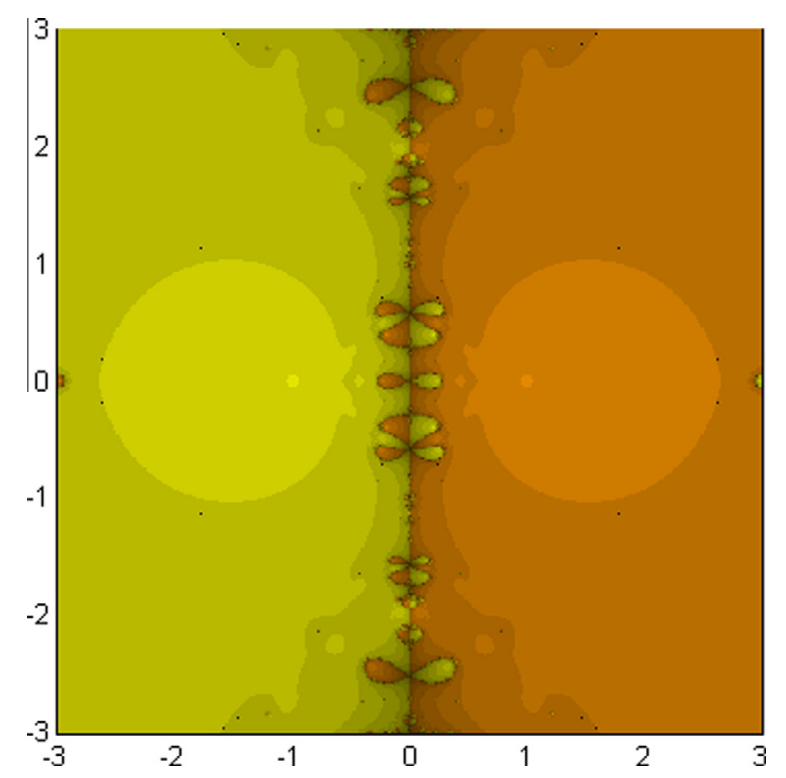

Fig. 6. Our method with $g=2.9, \gamma=-4, C=-4$, and any $\alpha$ and $A$ for the roots of the polynomial $z^{2}-1$.

$$
\begin{aligned}
& y_{n}=x_{n}-\frac{f\left(x_{n}\right)}{f^{\prime}\left(x_{n}\right)}, \\
& \tau_{n}=x_{n}-\left[\left(\frac{f\left(y_{n}\right)}{f\left(x_{n}\right)}\right)^{2}-\frac{f\left(x_{n}\right)}{f\left(y_{n}\right)-f\left(x_{n}\right)}\right] \frac{f\left(x_{n}\right)}{f^{\prime}\left(x_{n}\right)}, \\
& x_{n+1}=\tau_{n}-\left[\phi\left(\frac{f\left(y_{n}\right)}{f\left(x_{n}\right)}\right)+\frac{f\left(\tau_{n}\right)}{f\left(y_{n}\right)-a f\left(\tau_{n}\right)}+\frac{4 f\left(\tau_{n}\right)}{f\left(x_{n}\right)}\right] \frac{f\left(\tau_{n}\right)}{f^{\prime}\left(x_{n}\right)},
\end{aligned}
$$

where $\phi$ is an arbitrary real function satisfying the conditions

$$
\phi(0)=1, \quad \phi^{\prime}(0)=2, \quad \phi^{\prime \prime}(0)=4, \quad \phi^{\prime \prime \prime}(0)=-6 .
$$

We have taken $a=1$ and $\phi(t)=1+2 t+2 t^{2}-t^{3}$. 
Table 5

Average number of iterations per point.

\begin{tabular}{|c|c|c|c|c|c|c|}
\hline Case & Ex1 & Ex2 & EX3 & Ex4 & Ex5 & Total \\
\hline 1 & 3.2708 & 4.7640 & 7.5009 & 6.0247 & 11.6231 & 33.1835 \\
\hline 2 & 3.1197 & 4.3875 & 6.6839 & 5.8369 & 10.7186 & 30.7466 \\
\hline 3 & 3.2751 & 4.3391 & 5.6630 & 5.6329 & 8.2313 & 27.1414 \\
\hline 4 & 3.1885 & 4.1557 & 5.4185 & 5.8138 & 8.5328 & 27.1093 \\
\hline 5 & 3.1163 & 4.3463 & 6.0809 & 5.5301 & 9.9465 & 29.0201 \\
\hline 6 & 3.2751 & 4.3391 & 5.6630 & 5.6329 & 8.2313 & 27.1414 \\
\hline 7 & 3.1885 & 4.1557 & 5.4185 & 5.8138 & 8.5328 & 27.1093 \\
\hline 8 & 3.2452 & 5.0434 & 8.0473 & 5.9350 & 11.9265 & 34.1974 \\
\hline
\end{tabular}

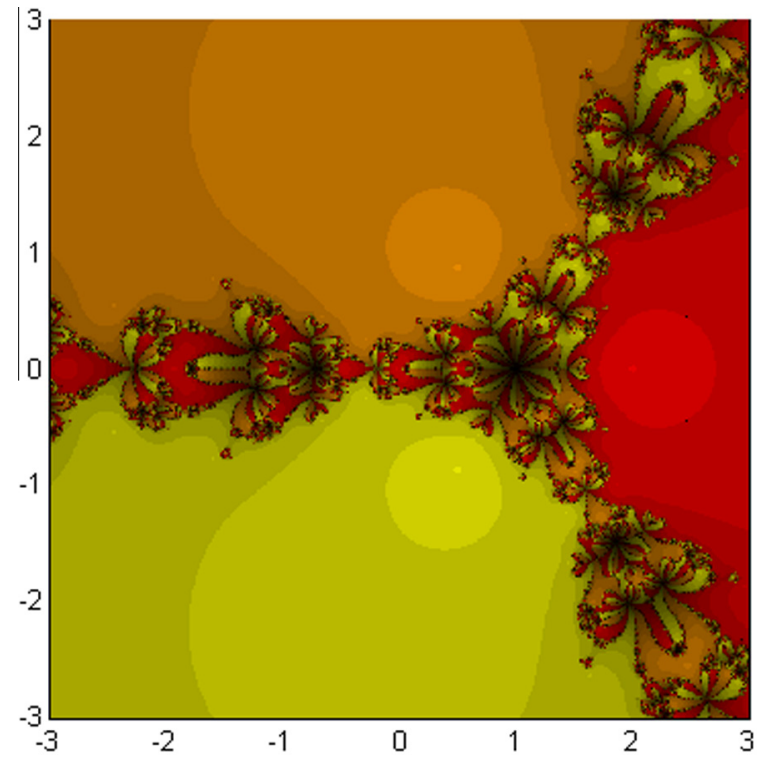

Fig. 7. Our method with $g=41 / 36, \gamma=2, C=-2 / 3, \alpha=1$, and $A=-3$ for the roots of the polynomial $(z-1)^{3}-1$.

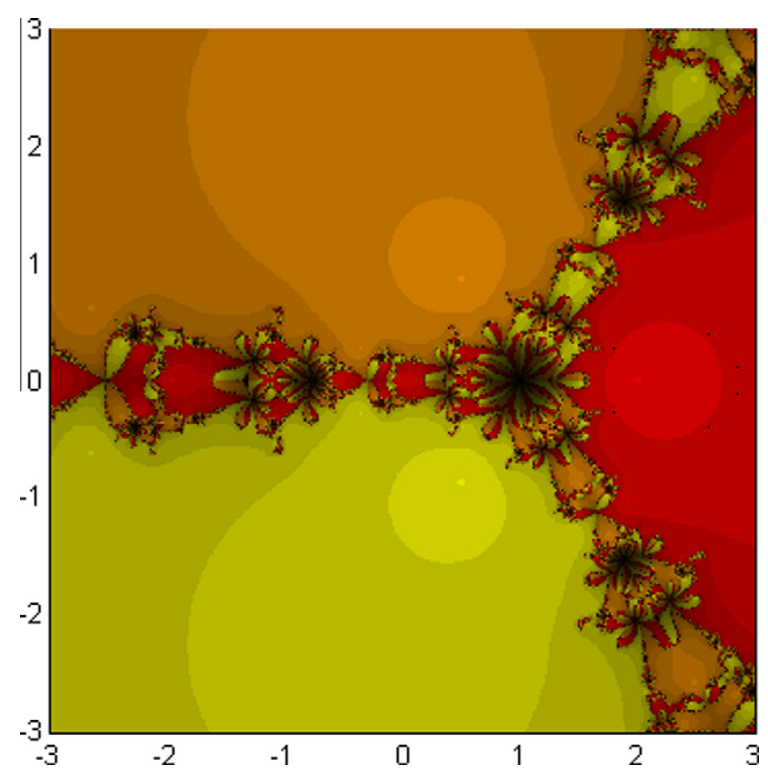

Fig. 8. Our method with $g=41 / 36, \gamma=-1, C=-2 / 3, \alpha=2$, and $A=1$ for the roots of the polynomial $(z-1)^{3}-1$. 


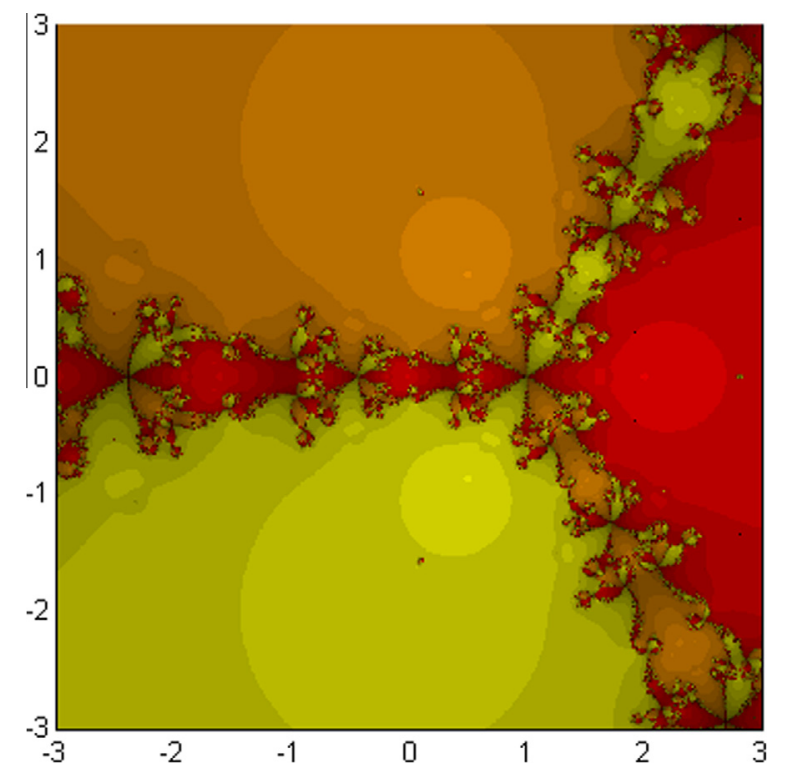

Fig. 9. Our method with $g=-4, \gamma=0, C=-4$, and any $\alpha$ and $A$ for the roots of the polynomial $(z-1)^{3}-1$.

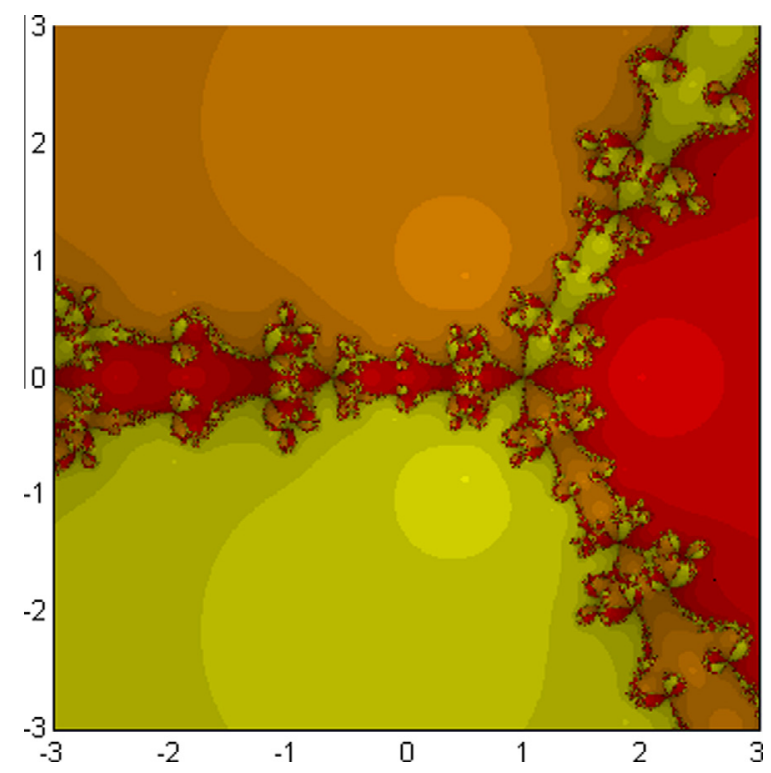

Fig. 10. Our method with $g=-4, \gamma=0, C=0$, and any $\alpha$ and $A$ for the roots of the polynomial $(z-1)^{3}-1$.

In Table 1 we presented the results for KT8 (21), HKT8 (22), HKN8 (24), N8 (26) with $\beta=2$, WM8 (28) and our new methods OM (OM1 is with $\alpha=1, A=-3, \gamma=2$ and OM2 is with $\alpha=2, A=1, \gamma=-1)$. In Table 2 we also presented the results for our methods OMN1 (case 3, see Table 3), OMN2 (case 4), OMN3 (case 5) and OMN6 (case 8). The number of iterations IT required to converge is given along with the value of the function at the last iteration $f\left(x_{*}\right)$. It can be observed that for most of the considered test functions our methods show as good performance as the other methods in their convergence speed and also have reasonable smallness of the residuals. In fact, in one case $\left(f_{8}\right)$ our methods converged even though WM8 diverged. We also found that in that example OMN6 diverged even though the other methods converged. We will show later that OMN6 is not a good choice. Therefore we can conclude that the new methods (OM1, OM2, OMN1-OMN3) are competitive with other eighth-order schemes being considered for solving nonlinear equations. 


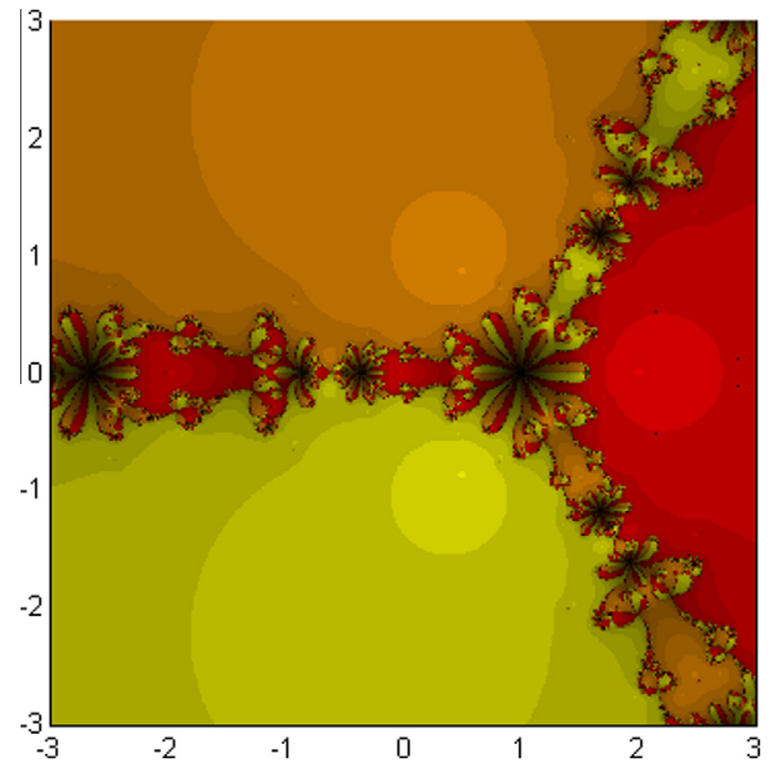

Fig. 11. Our method with $g=0, \gamma=-4, C=0$, and any $\alpha$ and $A$ for the roots of the polynomial $(z-1)^{3}-1$.

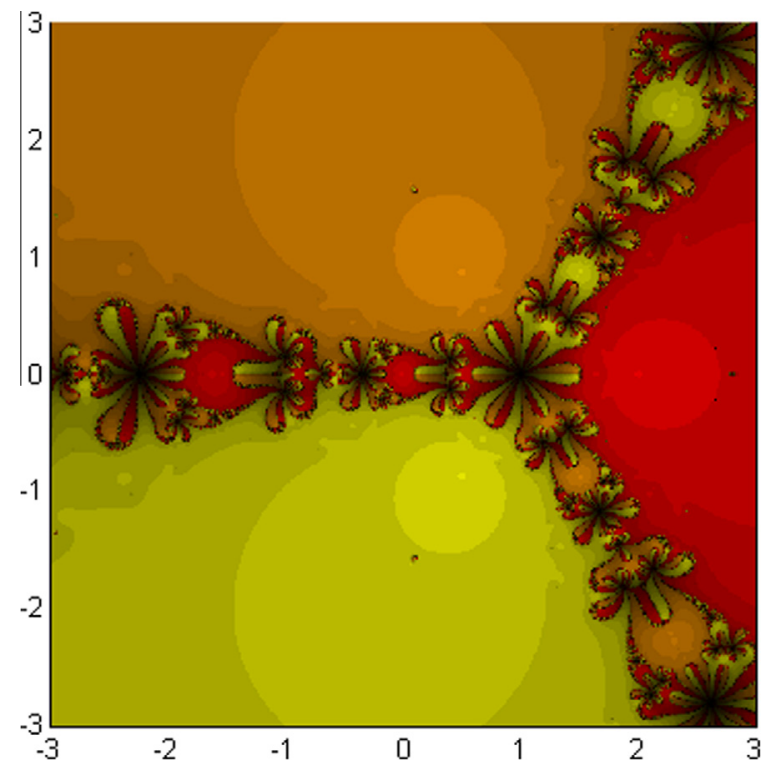

Fig. 12. Our method with $g=2.9, \gamma=-4, C=-4$, and any $\alpha$ and $A$ for the roots of the polynomial $(z-1)^{3}-1$.

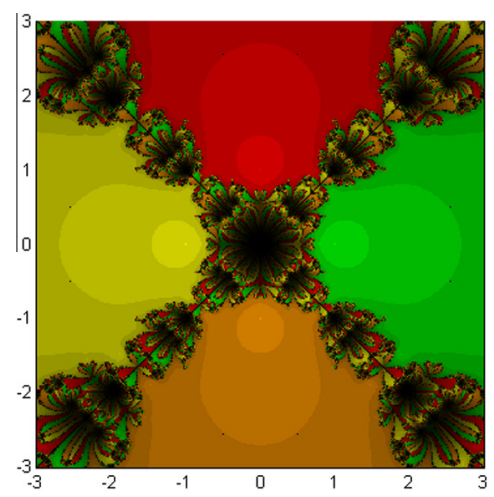

Fig. 13. Our method with $g=41 / 36, \gamma=2, C=-2 / 3, \alpha=1$, and $A=-3$ for the roots of the polynomial $z^{4}-1$. 


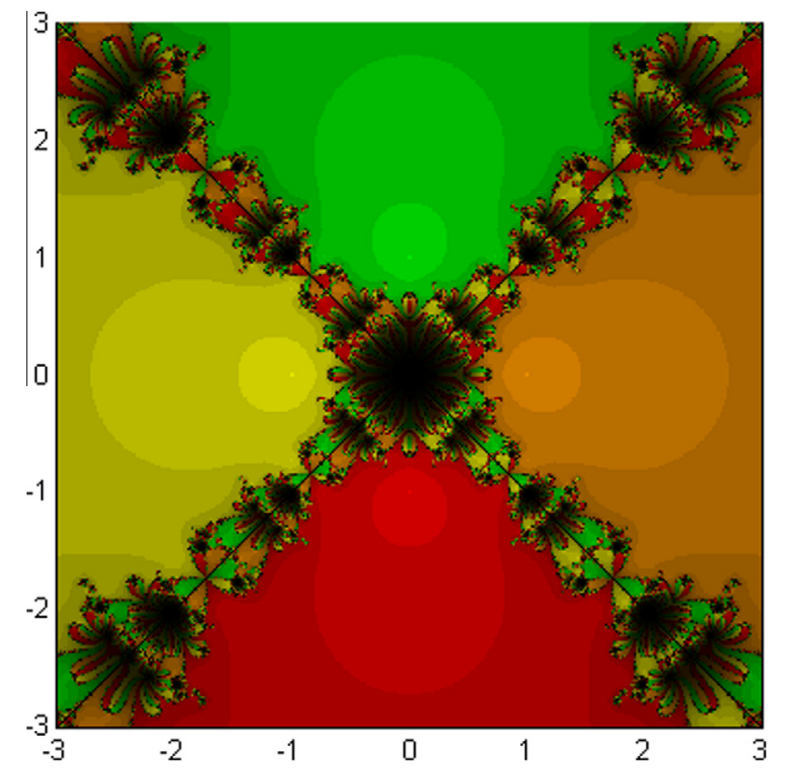

Fig. 14. Our method with $g=41 / 36, \gamma=-1, C=-2 / 3, \alpha=2$, and $A=1$ for the roots of the polynomial $z^{4}-1$.

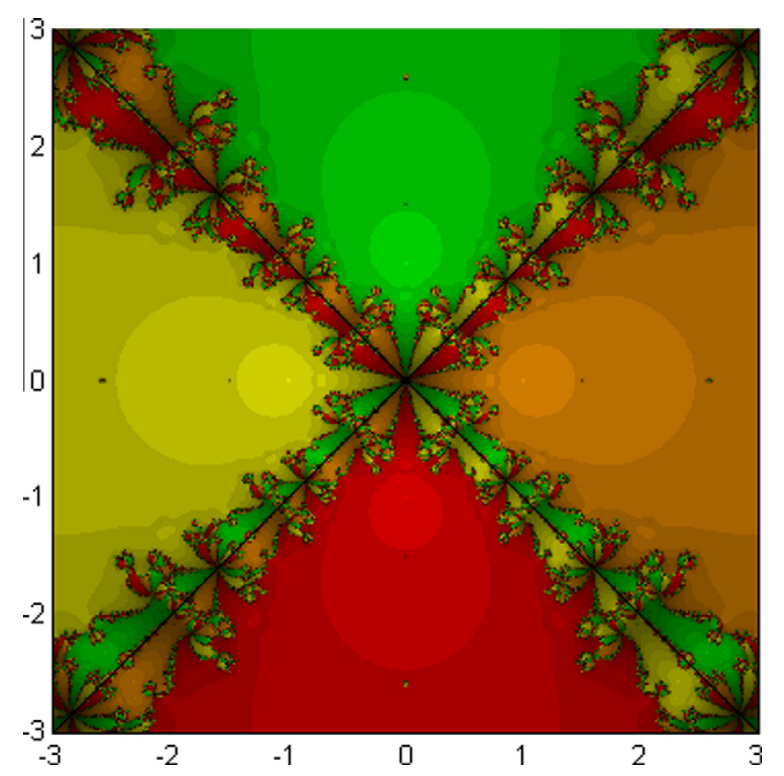

Fig. 15. Our method with $g=-4, \gamma=0, C=-4$, and any $\alpha$ and $A$ for the roots of the polynomial $z^{4}-1$.

In the next section, we analyze the basin of attraction of our eighth order family of methods to find out what is the best choice for the parameters. The idea of using basins of attraction was initiated by Stewart [9] and followed by the works of Amat et al. [10-13], Scott et al. [14], Chun et al. [15], Chicharro et al. [16], Cordero et al. [17], Neta et al. [18] and Chun et al. [19]. The only papers comparing basins of attraction for methods to obtain multiple roots is due to Neta et al. [20] and Neta and Chun [21-23].

\section{Basins of attraction}

In this section we give the basins of attraction of various members of the families OM and OMN. The 8 members are listed with their parameters in Table 3. The first 2 cases are of OM type and the last 6 cases are of OMN type. 


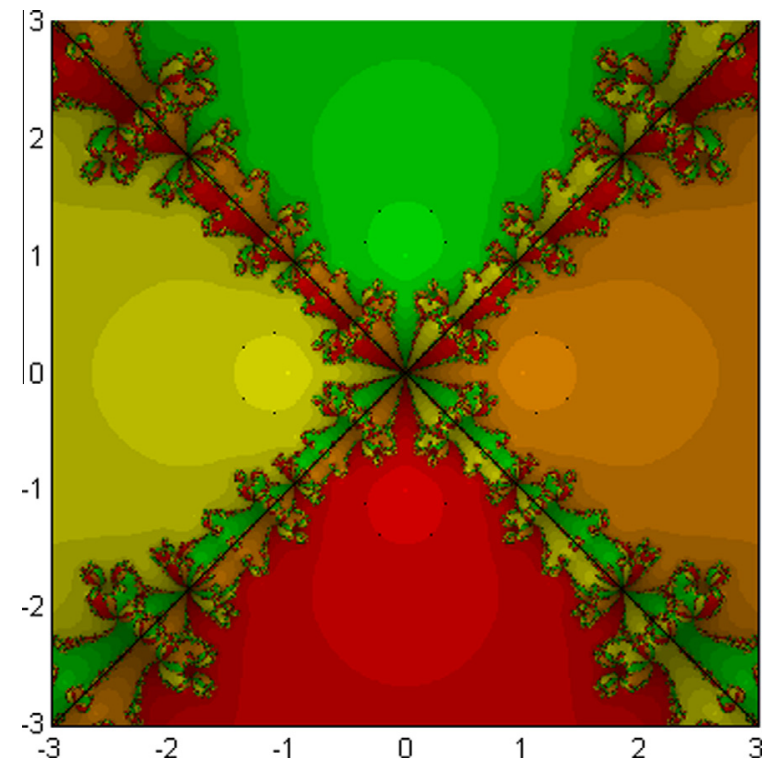

Fig. 16. Our method with $g=-4, \gamma=0, C=0$, and any $\alpha$ and $A$ for the roots of the polynomial $z^{4}-1$.

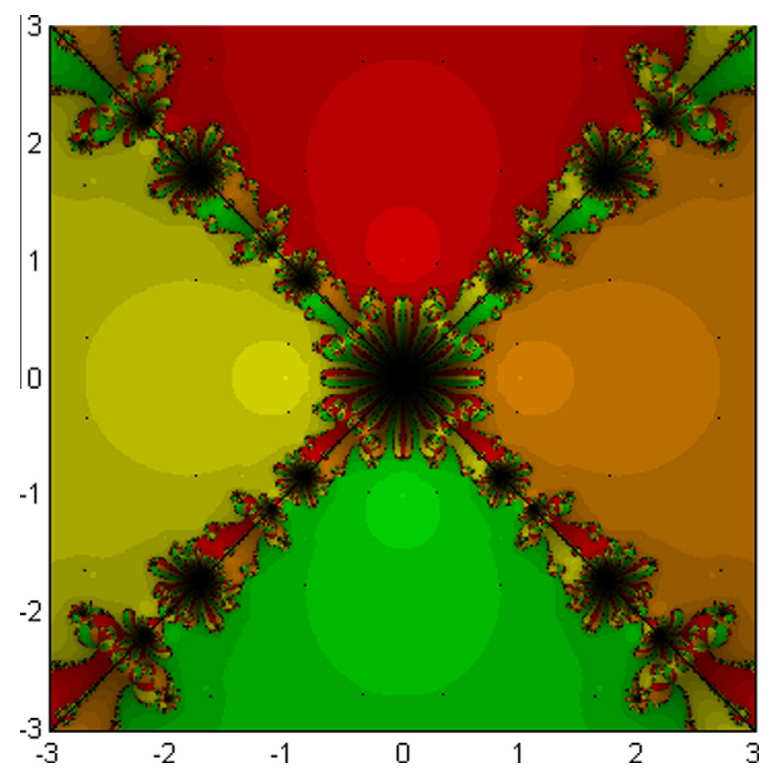

Fig. 17. Our method with $g=0, \gamma=-4, C=0$, and any $\alpha$ and $A$ for the roots of the polynomial $z^{4}-1$.

The first 2 cases were chosen so that we annihilate two terms in the error constant. The first of those was arbitrarily picked. In order to understand the choice of the parameters in the other cases we discuss the extraneous fixed points. In solving a nonlinear equation iteratively we are looking for fixed points which are zeros of the given nonlinear function. Many multipoint iterative methods have fixed points that are not zeros of the function of interest. Thus, it is imperative to investigate the number of extraneous fixed points, their location and their properties. In the family of methods described in this paper, the parameters $g, \gamma$, and $C$ can be chosen to position the extraneous fixed points on or close to the imaginary axis. This idea is due to Neta et al. [18] where they have shown an improvement in King's method by choosing the parameter that will position the extraneous fixed points on the imaginary axis. The second case was chosen so that we also have the extraneous fixed points as close as possible to the imaginary axis. Similarly, in the next 5 cases we have chosen the extraneous fixed 


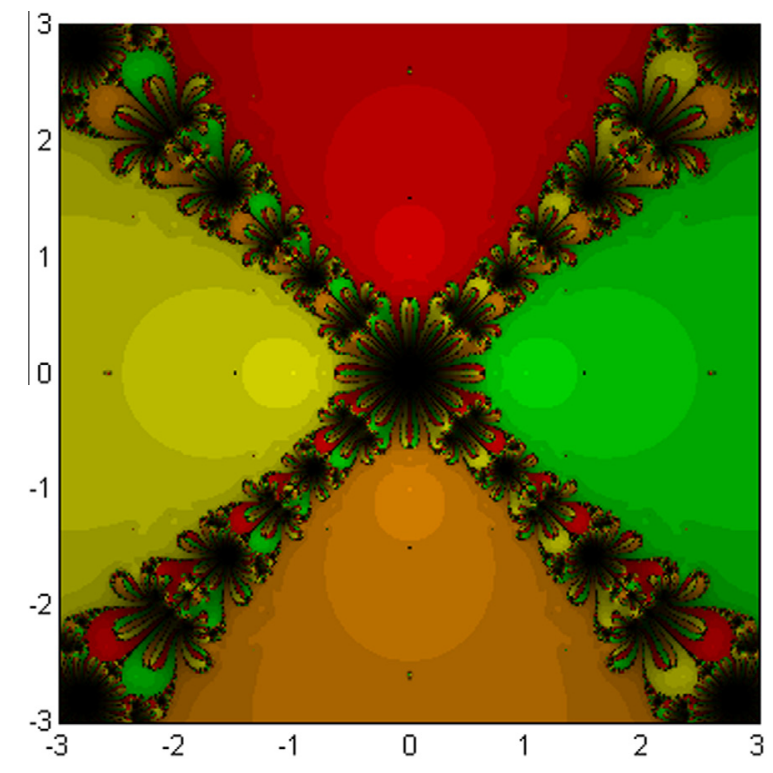

Fig. 18. Our method with $g=2.9, \gamma=-4, C=-4$, and any $\alpha$ and $A$ for the roots of the polynomial $z^{4}-1$.

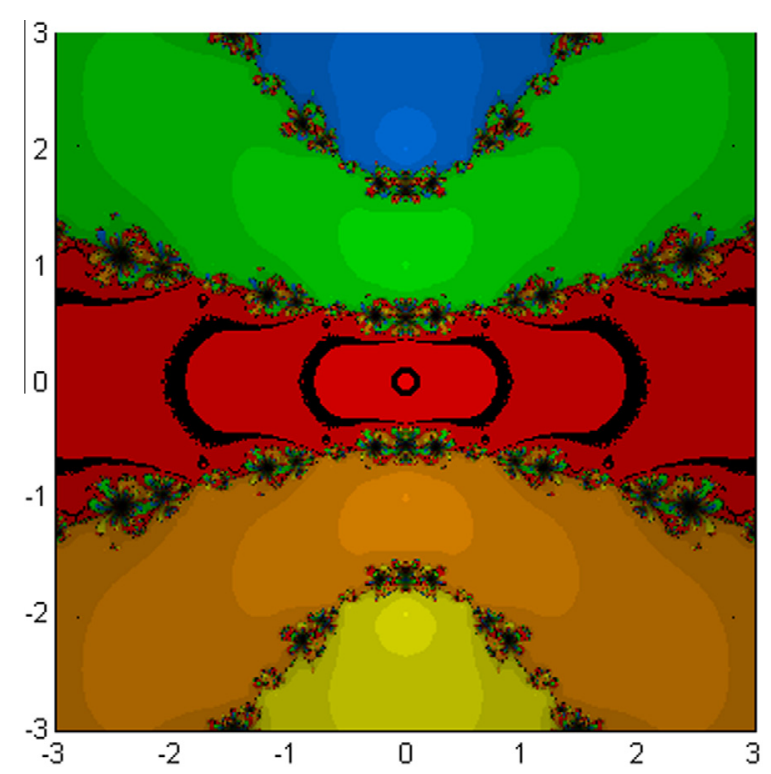

Fig. 19. Our method with $g=41 / 36, \gamma=2, C=-2 / 3, \alpha=1$, and $A=-3$ for the roots of the polynomial $z\left(z^{2}+1\right)\left(z^{2}+4\right)$.

points to be close to the imaginary axis. The last case violates that condition and one can see later that this violation increases the average number of iterations.

The eighth order family of methods discussed here can be written as

$$
x_{n+1}=x_{n}-\frac{f\left(x_{n}\right)}{f^{\prime}\left(x_{n}\right)} H_{f}\left(x_{n}\right),
$$

where

$$
H_{f}\left(x_{n}\right)=1+\frac{r_{n}}{\left(1-r_{n}\right)^{2}}+\frac{t_{n}}{\left(1-H\left(r_{n}\right) J\left(t_{n}\right) P\left(q_{n}\right)\right)^{2}} .
$$




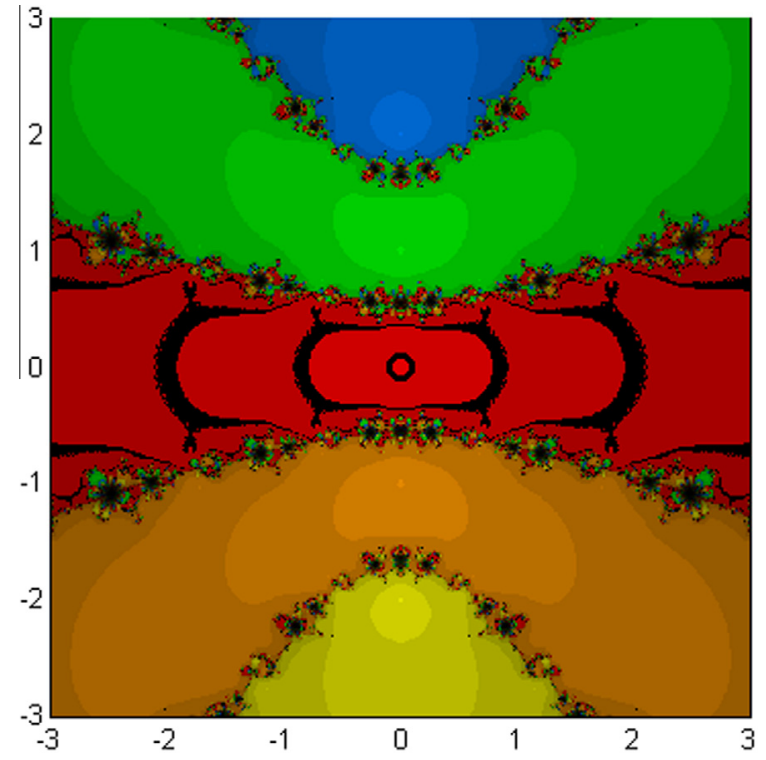

Fig. 20. Our method with $g=41 / 36, \gamma=-1, C=-2 / 3, \alpha=2$, and $A=1$ for the roots of the polynomial $z\left(z^{2}+1\right)\left(z^{2}+4\right)$.

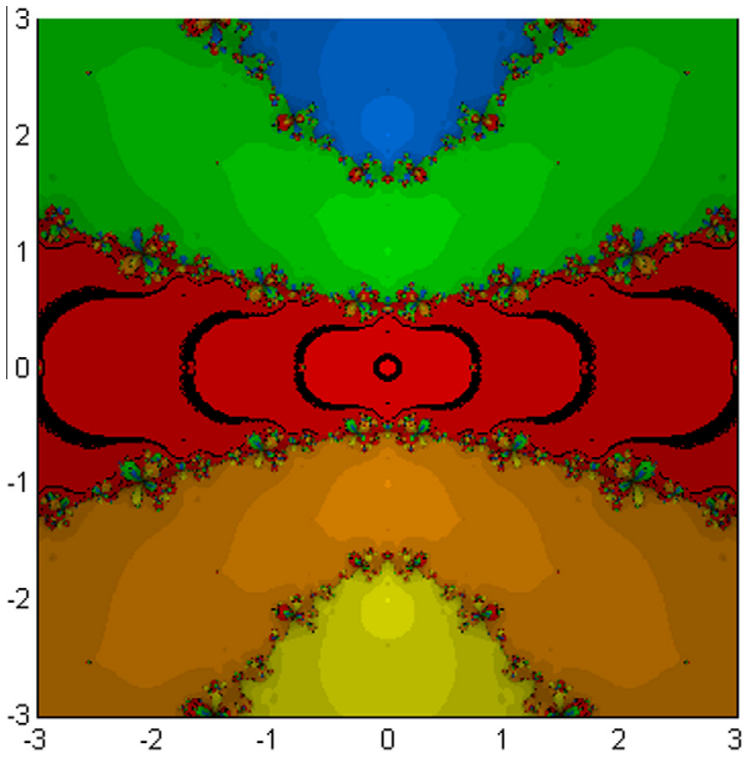

Fig. 21. Our method with $g=-4, \gamma=0, C=-4$, and any $\alpha$ and $A$ for the roots of the polynomial $z\left(z^{2}+1\right)\left(z^{2}+4\right)$.

Clearly the root $\xi$ of $f(x)$ is a fixed point of the method. The points $\alpha \neq \xi$ at which $H_{f}(\alpha)=0$ are also fixed points of the family. We have searched the parameter space and found that there are no point on the imaginary axis, but there are 5 cases with the smallest real part (these are denoted cases 3-7). To convince that this is a good choice we have taken a case where the real part of the extraneous roots is the largest (case 8). In Table 4 we have listed the number of extraneous fixed points (EFPs) and range of absolute value of the real parts of the EFPs for each case.

Example 1. In our first example we have used the polynomial $z^{2}-1$. The basins of attraction are given in Figs. 1-6. All the results are good. The best one is case 5 (Fig. 5) and the worst is case 3 (Fig. 3). The difference is barely noticeable. One can see it only when computing the average number of iterations per point (see Table 5.) All cases require between 3.1 and 3.3 iterations per point. Notice that cases 6 and 7 were not shown since we found that they yield identical results to cases 3 and 4 , respectively. 


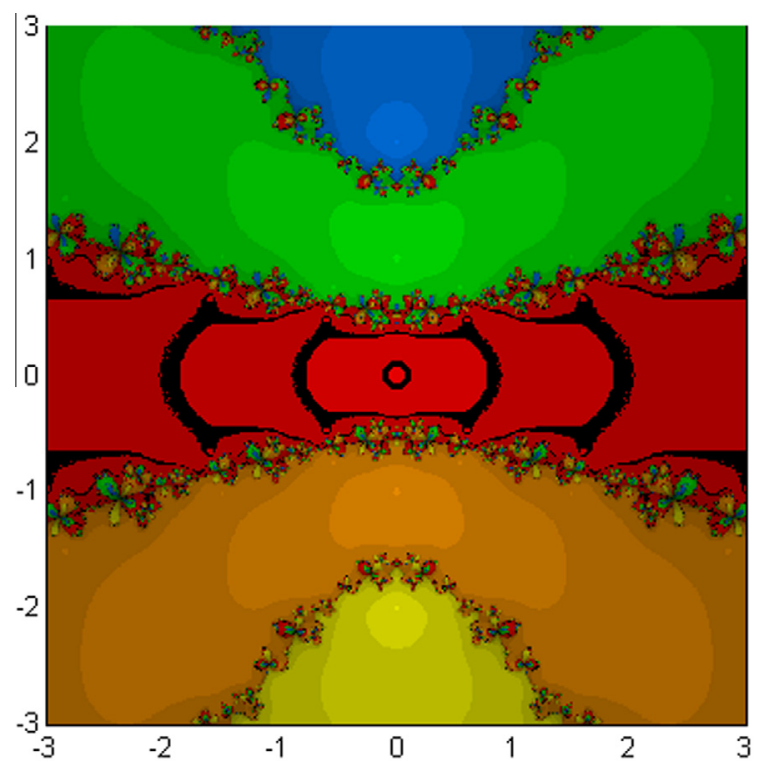

Fig. 22. Our method with $g=-4, \gamma=0, C=0$, and any $\alpha$ and $A$ for the roots of the polynomial $z\left(z^{2}+1\right)\left(z^{2}+4\right)$.

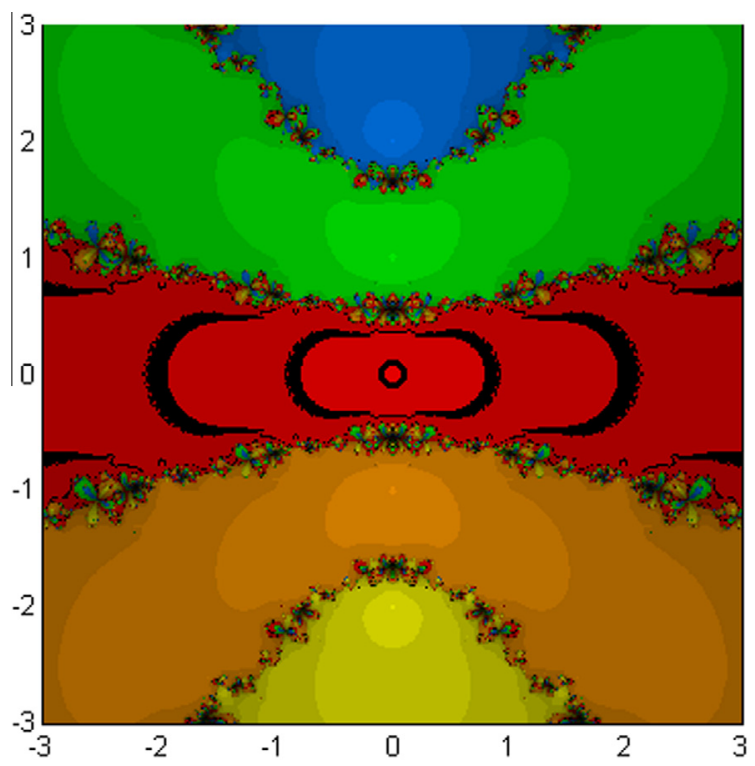

Fig. 23. Our method with $g=0, \gamma=-4, C=0$, and any $\alpha$ and $A$ for the roots of the polynomial $z\left(z^{2}+1\right)\left(z^{2}+4\right)$.

Example 2. In our next example we used the polynomial

$$
p_{2}(z)=(z-1)^{3}-1 .
$$

The basins are given in Figs. 7-12. In this case the best performer is case 4 (Fig. 10) and the worst is case 8 (Fig. 12).

Example 3. In our third example we have taken the polynomial

$$
p_{3}(z)=z^{4}-1 .
$$

The basins are given in Figs. 13-18. Again the best performer is case 4 (Fig. 16) and the worst is case 8 (Fig. 18). 


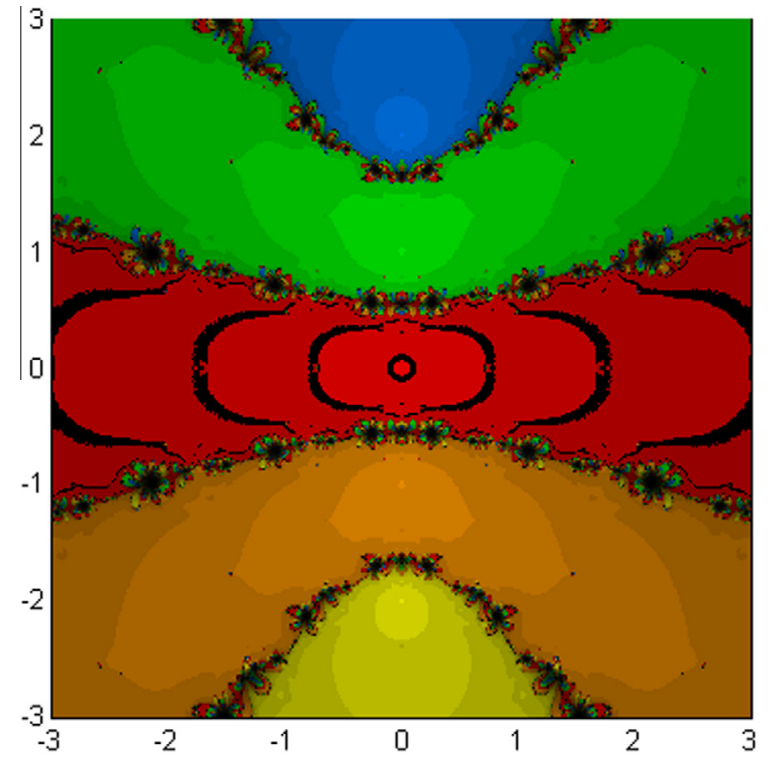

Fig. 24. Our method with $g=2.9, \gamma=-4, C=-4$, and any $\alpha$ and $A$ for the roots of the polynomial $z\left(z^{2}+1\right)\left(z^{2}+4\right)$.

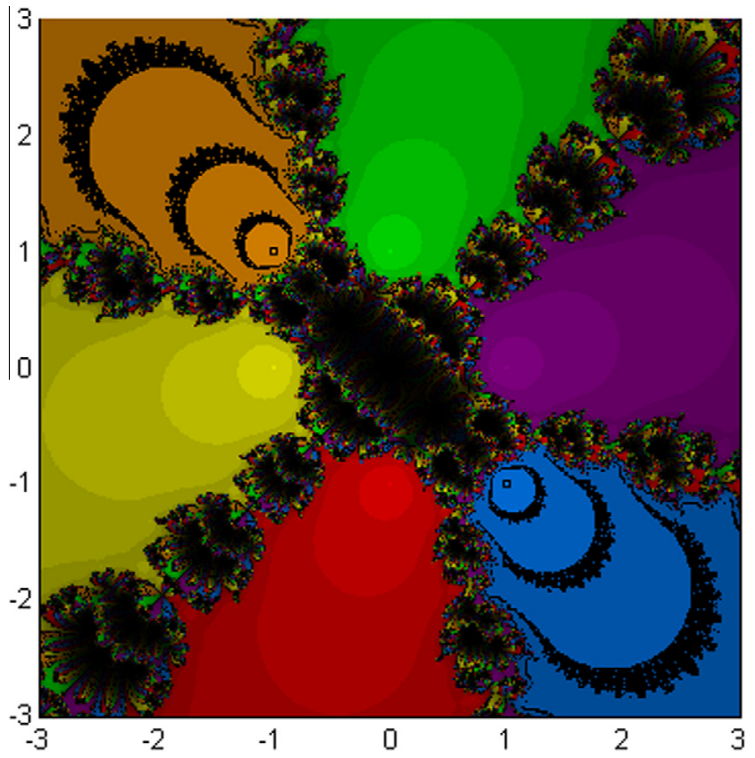

Fig. 25. Our method with $g=41 / 36, \gamma=2, C=-2 / 3, \alpha=1$, and $A=-3$ for the roots of the polynomial $\left(z^{2}-1\right)\left(z^{2}+1\right)\left(z^{2}+2 i\right)$.

Example 4. In our fourth example we have taken the polynomial

$$
p_{4}(z)=z\left(z^{2}+1\right)\left(z^{2}+4\right),
$$

whose roots are $0, \pm i, \pm 2 i$. The basins are given in Figs. 19-24. In this example the best is case 5 (Fig. 23) and the worst is case 1 (Fig. 19).

Example 5. In our last example we have taken the polynomial

$$
p_{5}(z)=\left(z^{2}-1\right)\left(z^{2}+1\right)\left(z^{2}+2 i\right)
$$




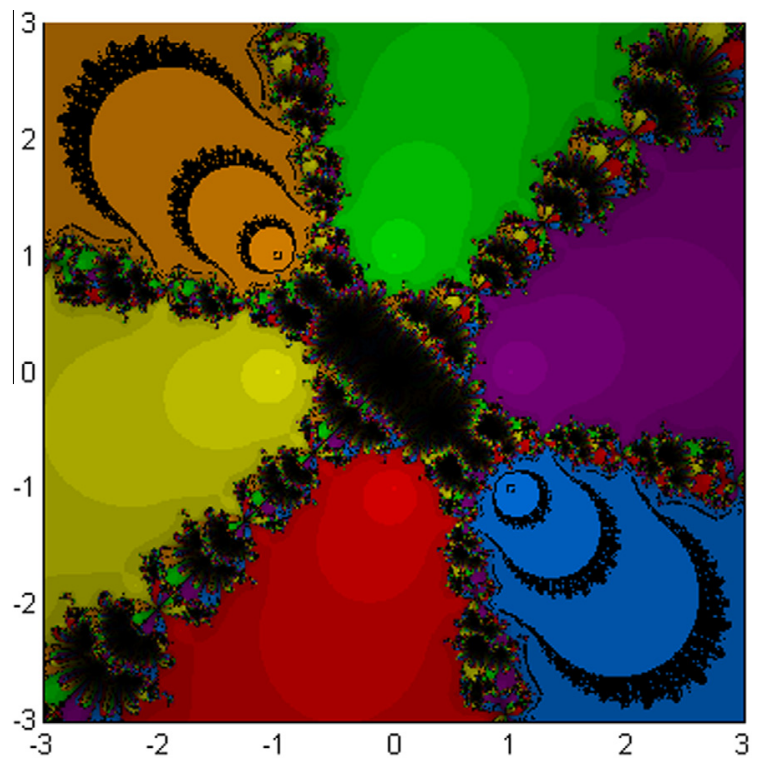

Fig. 26. Our method with $g=41 / 36, \gamma=-1, C=-2 / 3, \alpha=2$, and $A=1$ for the roots of the polynomial $\left(z^{2}-1\right)\left(z^{2}+1\right)\left(z^{2}+2 i\right)$.

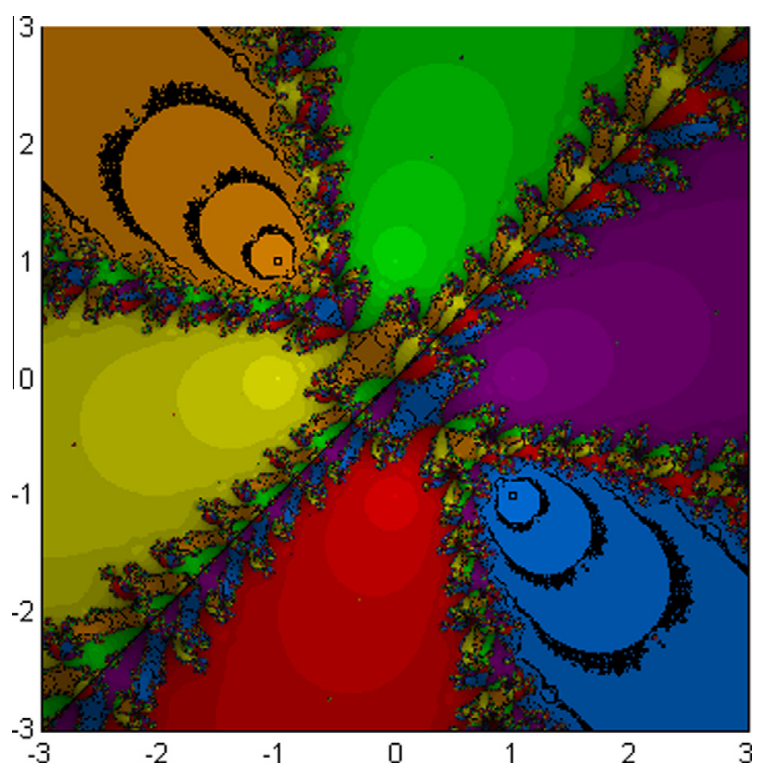

Fig. 27. Our method with $g=-4, \gamma=0, C=-4$, and any $\alpha$ and $A$ for the roots of the polynomial $\left(z^{2}-1\right)\left(z^{2}+1\right)\left(z^{2}+2 i\right)$.

whose roots are $\pm 1, \pm i,-1+i$, and $1-i$. The basins are given in Figs. 25-30. The best is case 3 (Fig. 28) and the worst is case 8 (Fig. 30).

Based on Table 5, the best case overall is case 4 for which $g=-4$ and $C=\gamma=0$ and the worst is case 8 for which $g=2.9$ and $C=\gamma=-4$. In general cases 3-5 (OMN) are better than cases 1-2(OM). Case 8 is also OMN but there we picked parameters that lead to largest (in absolute value) real part of the extraneous fixed points. On the other hand, cases $3-5$ have the smallest real part.

\section{Remarks.}

(1) Note that cases 3 and 6 yield identical results. Similarly cases 4 and 7 are identical. For this reason, we have not shown the results for cases 6 and 7 . 


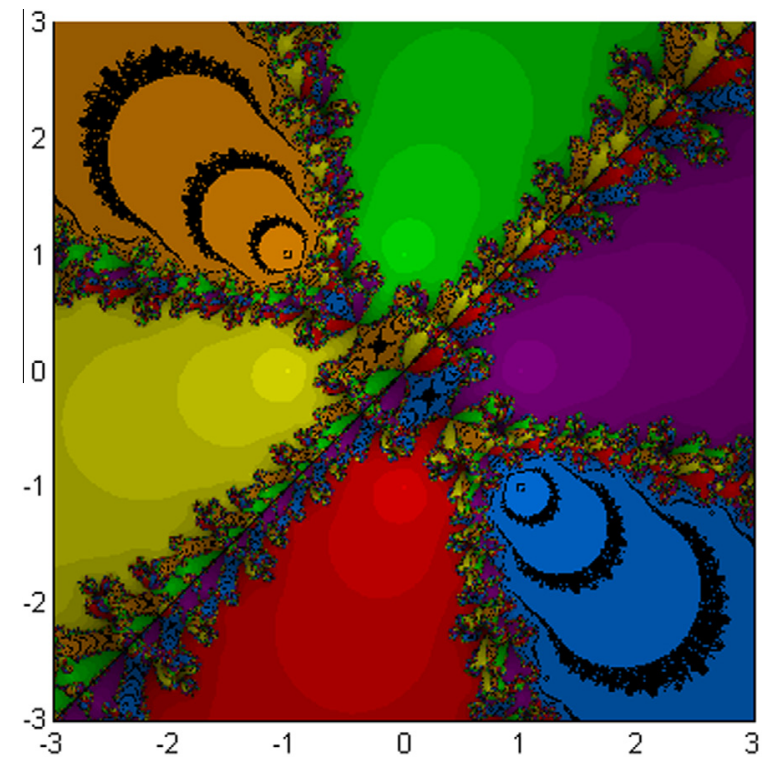

Fig. 28. Our method with $g=-4, \gamma=0, C=0$, and any $\alpha$ and $A$ for the roots of the polynomial $\left(z^{2}-1\right)\left(z^{2}+1\right)\left(z^{2}+2 i\right)$.

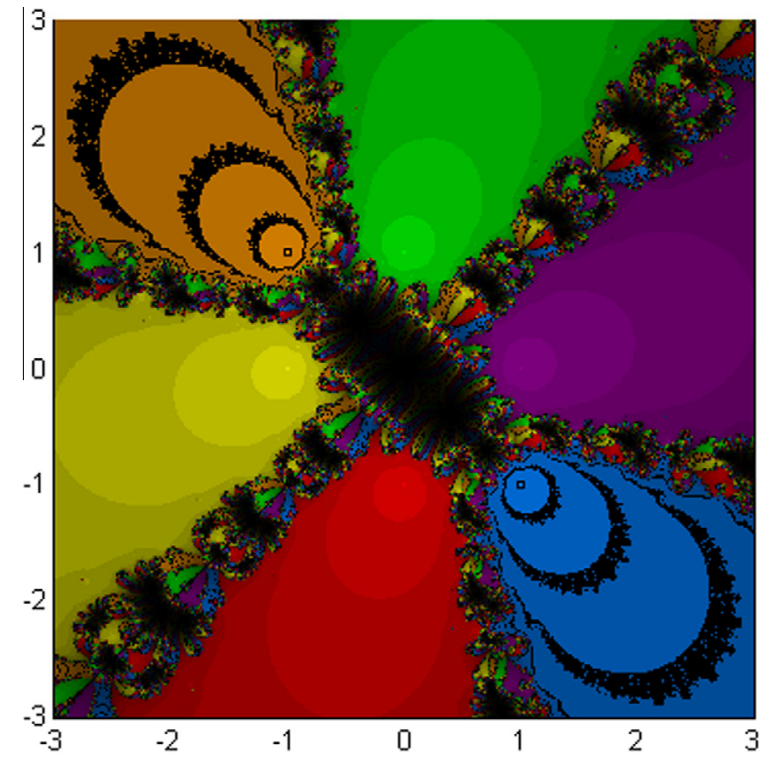

Fig. 29. Our method with $g=0, \gamma=-4, C=0$, and any $\alpha$ and $A$ for the roots of the polynomial $\left(z^{2}-1\right)\left(z^{2}+1\right)\left(z^{2}+2 i\right)$.

(2) Out of the first 2 cases, were we annihilated two terms in the error constant, the best is case 2 were we also chose the extraneous fixed points to be closest to the imaginary axis.

\section{Conclusions}

In this paper we have developed a new family of optimal eighth order iterative method. The scheme is optimal in the sense that it satisfies the Kung-Traub conjecture. We have compared several members of our family to existing optimal eighth order schemes and found that some members of our family are competitive. We have shown how to choose the parameters of the family to find the best members by evaluating all the extraneous fixed points. We have shown that the 


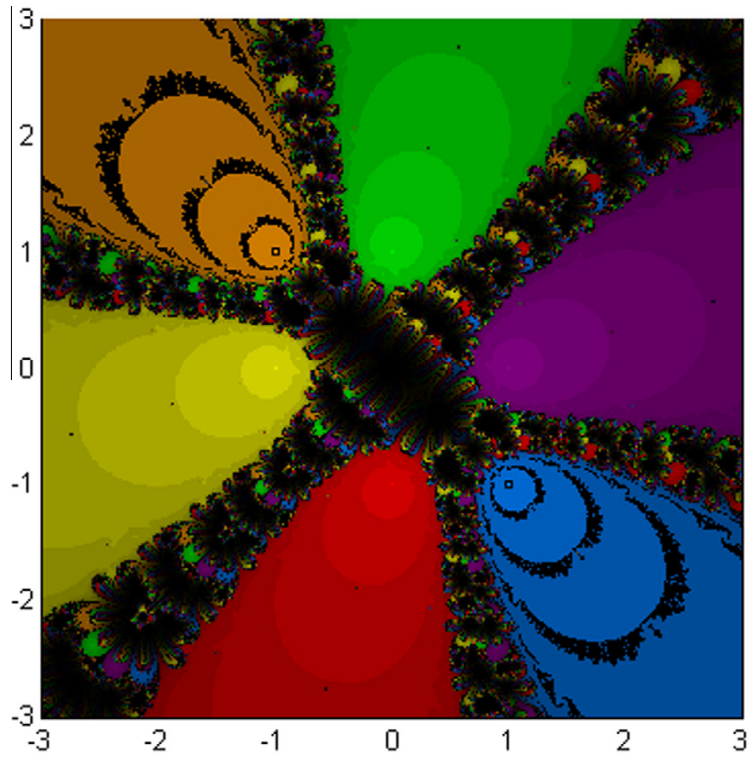

Fig. 30. Our method with $g=2.9, \gamma=-4, C=-4$, and any $\alpha$ and $A$ for the roots of the polynomial $\left(z^{2}-1\right)\left(z^{2}+1\right)\left(z^{2}+2 i\right)$.

best members have extraneous fixed point close to the imaginary axis. One member (case 8 ) for which this is not true was the worst perform.

\section{Acknowledgments}

This research was supported by Basic Science Research Program through the National Research Foundation of Korea (NRF) funded by the Ministry of Education (NRF-2013R1A1A2005012).

\section{References}

[1] J.F. Traub, Iterative Methods for the Solution of Equations, Chelsea Publishing Company, New York, 1977.

[2] M.S. Petković, B. Neta, L.D. Petković, J. Džunić, Multipoint Methods for Solving Nonlinear Equations, Elsevier, 2012.

[3] H.T. Kung, J.F. Traub, Optimal order of one-point and multipoint iterations, J. Assoc. Comput. Mach. 21 (1974) 643-651.

[4] C. Chun, B. Neta, A new sixth-order scheme for nonlinear equations, Appl. Math. Lett. 25 (2012) 185-189.

[5] B. Neta, C. Chun, M. Scott, Basins of attraction for optimal eighth order methods to find simple roots of nonlinear equations, Appl. Math. Comput. 227 (2014) 567-592.

[6] B. Neta, On a family of multipoint methods for nonlinear equations, Int. J. Comput. Math. 9 (1981) 353-361.

[7] R.F. King, A family of fourth-order methods for nonlinear equations, SIAM Numer. Anal. 10 (1973) 876-879.

[8] A.K. Maheshwari, A fourth-order iterative method for solving nonlinear equations, Appl. Math. Comput. 211 (2009) $383-391$.

[9] B.D. Stewart, Attractor basins of various root-finding methods (M.S. thesis), Naval Postgraduate School, Department of Applied Mathematics, Monterey, CA, June 2001.

[10] S. Amat, S. Busquier, S. Plaza, Iterative root-finding methods, unpublished report, 2004.

[11] S. Amat, S. Busquier, S. Plaza, Review of some iterative root-finding methods from a dynamical point of view, Scientia 10 (2004) 3-35.

[12] S. Amat, S. Busquier, S. Plaza, Dynamics of a family of third-order iterative methods that do not require using second derivatives, Appl. Math. Comput. 154 (2004) 735-746.

[13] S. Amat, S. Busquier, S. Plaza, Dynamics of the King and Jarratt iterations, Aeq. Math. 69 (2005) $212-2236$.

[14] M. Scott, B. Neta, C. Chun, Basin attractors for various methods, Appl. Math. Comput. 218 (2011) 2584-2599.

[15] C. Chun, M.Y. Lee, B. Neta, J. Džunić, On optimal fourth-order iterative methods free from second derivative and their dynamics, Appl. Math. Comput. 218 (2012) 6427-6438.

[16] F. Chircharro, A. Cordero, J.M. Gutiérrez, J.R. Torregrosa, Complex dynamics of derivative-free methods for nonlinear equations, Appl. Math. Comput. 219 (2013) 7023-7035.

[17] A. Cordero, J. García-Maimó, J.R. Torregrosa, M.P. Vassileva, P. Vindel, Chaos in King's iterative family, Appl. Math. Lett. 26 (2013) 842-848.

[18] B. Neta, M. Scott, C. Chun, Basin of attractions for several methods to find simple roots of nonlinear equations, Appl. Math. Comput. 218 (2012) 1054810556.

[19] C. Chun, B. Neta, Sujin Kim, On Jarratt's family of optimal fourth-order iterative methods and their dynamics, in press, http://dx.doi.org/10.1142/ S0218348X14500133.

[20] B. Neta, M. Scott, C. Chun, Basin attractors for various methods for multiple roots, Appl. Math. Comput. 218 (2012) $5043-5066$.

[21] B. Neta, C. Chun, On a family of Laguerre methods to find multiple roots of nonlinear equations, Appl. Math. Comput. 219 (2013) $10987-11004$.

[22] B. Neta, C. Chun, Basins of attraction for several optimal fourth order methods for multiple roots, Math. Comput. Simul. 103 (2014) 39-59.

[23] B. Neta, C. Chun, Basins of attraction for Zhou-Chen-Song fourth order family of methods for multiple roots, Math. Comput. Simul., submitted for publication. 